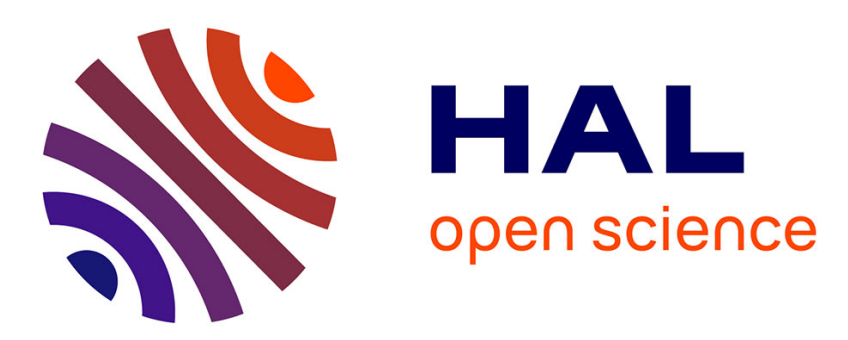

\title{
Connecting dispersion models and wall temperature prediction for laminar and turbulent flows in channels
}

Olivier Grégoire, Marie Drouin, Olivier Simonin

\section{To cite this version:}

Olivier Grégoire, Marie Drouin, Olivier Simonin. Connecting dispersion models and wall temperature prediction for laminar and turbulent flows in channels. International Journal of Heat and Mass Transfer, 2012, vol. 55, pp. 3100-3113. 10.1016/j.ijheatmasstransfer.2012.02.011 . hal-00914632

\section{HAL Id: hal-00914632 \\ https://hal.science/hal-00914632}

Submitted on 5 Dec 2013

HAL is a multi-disciplinary open access archive for the deposit and dissemination of scientific research documents, whether they are published or not. The documents may come from teaching and research institutions in France or abroad, or from public or private research centers.
L'archive ouverte pluridisciplinaire HAL, est destinée au dépôt et à la diffusion de documents scientifiques de niveau recherche, publiés ou non, émanant des établissements d'enseignement et de recherche français ou étrangers, des laboratoires publics ou privés. 


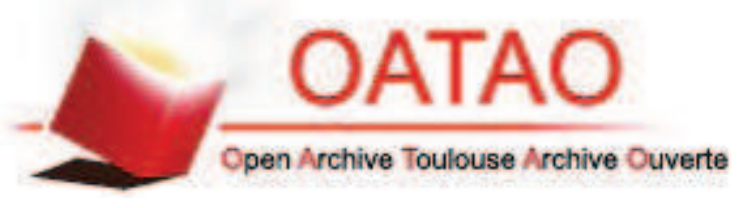

Open Archive TOULOUSE Archive Ouverte (OATAO)

OATAO is an open access repository that collects the work of Toulouse researchers and makes it freely available over the web where possible.

This is an author-deposited version published in : http://oatao.univ-toulouse.fr/ Eprints ID : 10363

To link to this article : DOI:10.1016/j.ijheatmasstransfer.2012.02.011 http://dx.doi.org/10.1016/j.ijheatmasstransfer.2012.02.011

To cite this version : Grégoire, Olivier and Drouin, Marie and Simonin, Olivier Connecting dispersion models and wall temperature prediction for laminar and turbulent flows in channels. (2012) International Journal of Heat and Mass Transfer, vol. 55 .pp. 3100-3113. ISSN 0017-9310

Any correspondance concerning this service should be sent to the repository administrator: staff-oatao@,1istes-diff.inp-toulouse.fr 


\title{
Connecting dispersion models and wall temperature prediction for laminar and turbulent flows in channels
}

\author{
O. Grégoire ${ }^{\mathrm{a}, *}$, M. Drouin ${ }^{\mathrm{b}}$, O. Simonin ${ }^{\mathrm{c}}$ \\ ${ }^{a}$ STXN, 25 rue Leblanc, 75015 Paris, France \\ ${ }^{\mathrm{b}}$ CEA Saclay, DEN/DANS/DM2S/SFME/LETR, 91191 Gif-sur-Yvette, France \\ ${ }^{\mathrm{C}}$ Université de Toulouse, CNRS; IMFT; Allée du Professeur Camille Soula, 31400 Toulouse, France
}

\section{Keywords:}

Heat transfer

Taylor dispersion

Double averaging

Turbulence

\begin{abstract}
A B S T R A C T
In a former paper, Drouin et al. [6] proposed a model for dispersion phenomena in heated channels that works for both laminar and turbulent regimes. This model, derived according to the double averaging procedure, leads to satisfactory predictions of mean temperature. In order to derive dispersion coefficients, the so called "closure problem" was solved, which gave us access to the temperature deviation at sub filter scale. We now propose to capitalize on this useful information in order to connect dispersion modeling to wall temperature prediction. As a first step, we use the temperature deviation modeling in order to connect wall to mean temperatures within the asymptotic limit of well established pipe flows. Since temperature in wall vicinity is mostly controlled by boundary conditions, it might evolve according to different time and length scales than averaged temperature. Hence, this asymptotic limit provides poor prediction of wall temperature when flow conditions encounter fast transients and stiff heat flux gradients. To overcome this limitation we derive a transport equation for temperature deviation $\left(T_{w}-\left\langle\bar{T}_{f}\right\rangle_{f}\right)$. The resulting two-temperature model is then compared with fine scale simulations used as reference results. Wall temperature predictions are found to be in good agreement for various Prandtl and Reynolds numbers, from laminar to fully turbulent regimes and improvement with respect to classical models is noticeable.
\end{abstract}

\section{Introduction}

Design, optimization and safety analysis of large heating devices such as heat exchangers or nuclear reactor cores are major concerns for many engineers. Those studies rely heavily upon flows and heat exchanges modeling. Indeed, considering the geometrical complexity and the size of such systems, it is not possible, to calculate the details of velocity and temperature profiles in each sub-channel. However, the primary interest for industrial purpose is not the details of the flow, but rather the description on a large scale of mean flow quantities and heat transfer properties. Such a macroscopic description may be obtained by applying up-scaling methods $[9,15,20,21]$. Doing so, a nuclear reactor core, for instance, can be described in an homogenized way (porous approach) by means of a spatial filter [1,19]. This averaging procedure leads to modified equations for mean flow variables, with additional contributions that account for small scale phenomena, mainly boundary layers interactions with solids. Actually, such heating devices might be seen as spatially periodic and anisotropic porous media,

\footnotetext{
* Corresponding author. Tel.: +33 164502743; fax: +33 164501307

E-mail address: olivier.gregoire@cea.fr (O. Grégoire).
}

with the additional difficulty that flows may achieve any regime, from laminar to highly turbulent, within the pores.

In a former article [6], a complete macroscopic mean temperature model for flows in stratified porous media has been presented. Correlations for scalar and temperature dispersion modeling in rectangular, circular and annular pipes have been established and assessed thanks to comparisons with fine scale simulation results. Now, we focus on wall temperature modeling, or, in other words, on heat exchange modeling. The most classical heat exchange coefficients do not account for transients flows or non uniform heat fluxes since they are based upon the assumption that flows are fully established. It is well known, for instance, that heat exchange in pipes inlet region are poorly predicted and ad hoc modifications of models are generally used [10]. During a fast transient or when heat fluxes encounter large gradients, flows cannot be considered established anymore. Boundary layers and bulk flow do not react simultaneously to those strong perturbations and are, in a sense, out of phase. Since dispersion modeling relies on the analysis of spatial deviations of flow quantities (the so-called "closure problem") [2], it appears to be a natural way to account for those unbalances.

In this work, we propose to connect dispersion modeling to heat transfer and averaged wall temperature modeling for forced 


\begin{tabular}{|c|c|c|c|}
\hline \multicolumn{4}{|c|}{ Nomenclature } \\
\hline$A_{f}$ & interface between solid and fluid phases $\left(\mathrm{m}^{2}\right)$ & $\delta_{w}$ & Dirac delta function associated to the walls $\left(\mathrm{m}^{-1}\right)$ \\
\hline $\mathcal{D}^{A}$ & thermal active dispersion vector $(\mathrm{m})$ & $\Delta T$ & wall to mean temperature gap $(\mathrm{K})$ \\
\hline $\mathcal{D}^{P}$ & thermal passive dispersion tensor $\left(\mathrm{m}^{2} \mathrm{~s}^{-1}\right)$ & $\Delta V$ & representative elementary volume (REV) $\left(\mathrm{m}^{3}\right)$ \\
\hline$C_{p}$ & Specific heat capacity $\left(\mathrm{J} \mathrm{kg}^{-1} \mathrm{~K}^{-1}\right)$ & $\Delta V_{f}$ & fluid volume included in the $\operatorname{REV}\left(\mathrm{m}^{3}\right)$ \\
\hline$D_{h}$ & hydraulic diameter of the pores (m) & $\zeta$ & active dispersion function $(\mathrm{s})$ \\
\hline$e_{1,2}$ & thickness of the central and near-wall layers & $\eta_{j}$ & passive dispersion function (m) \\
\hline$f_{w}$ & friction coefficient & $v_{f}$ & kinematic viscosity of the fluid $\left(\mathrm{m}^{2} \mathrm{~s}^{-1}\right)$ \\
\hline$n_{i}$ & $\begin{array}{l}\text { ith component of the interface normal vector, pointing } \\
\text { towards the solid phase }\end{array}$ & $\begin{array}{l}v_{t} \\
\rho\end{array}$ & $\begin{array}{l}\text { turbulent kinematic viscosity }\left(\mathrm{m}^{2} \mathrm{~s}^{-1}\right) \\
\text { density of the fluid }\left(\mathrm{kg} \mathrm{m}^{-3}\right)\end{array}$ \\
\hline $\mathrm{Pe}$ & Péclet number $\left(U D_{h} / \alpha_{f}=R e P r\right)$ & $\phi$ & porosity \\
\hline $\operatorname{Pr}$ & Prandtl number $\left(v_{f} \mid \alpha_{f}\right)$ & $\Phi$ & wall heat flux \\
\hline $\operatorname{Pr}_{t}$ & turbulent Prandtl number $\left(v_{t} / \alpha_{t}\right)$ & $\Sigma$ & solid surface \\
\hline $\operatorname{Re}$ & Reynolds number $\left(U D_{h} / v_{f}\right)$ & $\Sigma_{2,1}$ & surface delimiting inner and outer region \\
\hline$R$ & radius of the pipe & & \\
\hline REV & Representative Elementary Volume & \multicolumn{2}{|c|}{ Other symbols } \\
\hline$\Omega$ & volume of a REV & - & statistical average \\
\hline$\Omega_{1}$ & volume of the central region of the REV & $\therefore$ & fluctuation from the statistical average \\
\hline$\Omega_{2}$ & volume of the near-wall region of the REV & \langle\rangle & volume average \\
\hline$T_{f}$ & fluid temperature & \langle\rangle$_{f}$ & fluid volume average \\
\hline$T_{1,2}$ & $\begin{array}{l}\text { fluid temperature averaged over central and wall re- } \\
\text { gions, respectively }\end{array}$ & \langle\rangle$_{1,2}$ & $\begin{array}{l}\text { fluid volume average over central and wall regions of } \\
\text { the flow, respectively }\end{array}$ \\
\hline$u_{1,2}$ & $\begin{array}{l}\text { fluid velocity averaged over central and wall regions, } \\
\text { respectively }\end{array}$ & $\begin{array}{l}\delta \\
. *\end{array}$ & $\begin{array}{l}\text { deviation from the fluid volume average } \\
\text { dimensionless quantity }\end{array}$ \\
\hline & friction velocity $\left(\mathrm{m} \mathrm{s}^{-1}\right)$ & $\cdot f$ & fluid \\
\hline & & & bulk \\
\hline \multicolumn{2}{|c|}{ Greek symbols } & w & wall \\
\hline$\alpha_{f}$ & thermal diffusivity of the fluid $\left(\mathrm{m}^{2} \mathrm{~s}^{-1}\right)$ & $\cdot t$ & turbulent \\
\hline$\alpha_{t}$ & turbulent thermal diffusivity $\left(\mathrm{m}^{2} \mathrm{~s}^{-1}\right)$ & & \\
\hline & macroscopic turbulent thermal diffusivity $\left(\mathrm{m}^{2} \mathrm{~s}^{-1}\right)$ & & \\
\hline$\lambda_{f}$ & thermal conductivity of the fluid $(\mathrm{W} / \mathrm{m} / \mathrm{K})$ & & \\
\hline
\end{tabular}

convection flows in pipes. In Section 2, the averaging procedure and the derivation and closure of the macroscopic mean temperature equation are recalled. In Section 3, we show how it is possible to connect the temperature deviation modeling embedded in porous media approach with classical heat exchange models. Since temperature in wall vicinity is mostly controlled by boundary conditions, it might evolve according to different time and length scales than averaged temperature. Hence the resulting algebraic closure must be seen as an asymptotic limit consistent with both porous media modeling and classical heat exchange modeling for smooth flows.

For flows exhibiting large or rapid variations of boundary conditions, thermal unbalance might reach a high level so other time and length scales have to be taken into account. To overcome the limitation of algebraic models to represent such an effect, a method to derive a balance equation for wall temperature model is exposed in Section 4. New contributions in this equation are closed consistently with the algebraic limit of the porous model presented in Section 3. The resulting two-temperature model (averaged and wall temperatures) is finally assessed by detailed comparisons with fine scale simulation results and classical heat exchange models.

\section{Macroscopic temperature equation}

We recall in this section the averaging procedure and the derivation and closure of the macroscopic mean temperature equation. Since flows that are considered in this work can be turbulent, a statistical average operator, denoted “".", is used to handle the random character of turbulence. Our aim is to develop a spatially homogenized modeling of these flows, so we also apply a spatial filter. The spatial average operator used to derive a macroscale model is denoted " $\langle\cdot\rangle f$ ". For each average, any quantity $\xi$ may be split into mean and fluctuating components as

$\xi=\bar{\xi}+\xi^{\prime}=\langle\xi\rangle_{f}+\delta \xi$,

and one can write

$\xi=\langle\bar{\xi}\rangle_{f}+\left\langle\xi^{\prime}\right\rangle_{f}+\delta \bar{\xi}+\delta \xi^{\prime}$.

Statistical and spatial average properties are summarized in Drouin et al. [6]. The order of application for those averages is discussed in Pinson et al. [13].

In this study, incompressible and undilatable, single phase flows in saturated, rigid porous media are considered. Fluid properties (density, viscosity, heat capacity) and the porosity of the medium are assumed constant. Finally, we shall assume that thermal interactions with solids reduce to an external forcing for the fluid temperature. Under those hypothesis, macroscopic conservation of mass equation reads [13]:

$\frac{\partial\left\langle\bar{u}_{i}\right\rangle_{f}}{\partial x_{i}}=0$

Considering the thermal boundary condition on the wall $\mathcal{A}_{f}$ for statistically averaged temperature

$\alpha_{f} \frac{\partial \bar{T}_{f}}{\partial x_{i}} n_{i}=\frac{\bar{\Phi}}{\left(\rho C_{p}\right)_{f}} \quad$ on $\mathcal{A}_{f}$

and under the first gradient approximation for turbulent heat flux:

$\overline{u_{i}^{\prime} T_{f}^{\prime}}=-\alpha_{t} \frac{\partial \bar{T}_{f}}{\partial x_{i}}$,

Drouin et al. [6] (see Section 3) derived the following equation for mean temperature: 


$$
\begin{gathered}
\phi \frac{\partial\left\langle\bar{T}_{f}\right\rangle_{f}}{\partial t}+\frac{\partial}{\partial x_{i}}\left(\phi\left\langle\bar{u}_{i}\right\rangle_{f}\left\langle\bar{T}_{f}\right\rangle_{f}\right)=-\frac{\partial}{\partial x_{i}} \underbrace{\phi\left\langle\overline{u_{i}^{\prime} T_{f}^{\prime}}\right\rangle_{f}}_{\begin{array}{c}
\text { macroscopic } \\
\text { turbulent } \\
\text { heat flux }
\end{array}}+\frac{\partial}{\partial x_{i}}\left(\phi \alpha_{f}\right. \\
+\underbrace{\phi\left\langle\alpha_{f} \frac{\partial \bar{T}_{f}}{\partial x_{i}} n_{i} \delta_{w}\right\rangle_{f}}_{\begin{array}{c}
\text { wall heat } \\
\text { transfer }
\end{array}}+\underbrace{\frac{\partial}{\partial x_{i}} \phi\left\langle\alpha_{f} \delta \bar{T}_{f} n_{i} \delta_{w}\right\rangle_{f}}_{\text {tortuosity }}-\underbrace{\frac{\partial}{\partial x_{i}} \phi\left\langle\delta \bar{u}_{i} \delta \bar{T}_{f}\right\rangle_{f}}_{\begin{array}{c}
\text { thermal } \\
\text { dispersion }
\end{array}}
\end{gathered}
$$

Macroscopic turbulent heat flux can be modeled by means of a macroscopic turbulent thermal diffusivity $\alpha_{t \phi}[11,12]$ :

$$
\left\langle\overline{u_{i}^{\prime} T_{f}^{\prime}}\right\rangle_{f}=-\alpha_{t \phi} \frac{\partial\left\langle\bar{T}_{f}\right\rangle_{f}}{\partial x_{i}}=-\frac{v_{t \phi}}{\operatorname{Pr}_{t}} \frac{\partial\left\langle\bar{T}_{f}\right\rangle_{f}}{\partial x_{i}} .
$$

Macroscopic turbulent viscosity models for flows in pipes are detailed in Grégoire [8]. Thanks to Eq. (4), wall heat exchange reads

$\left\langle\alpha_{f} \frac{\partial \bar{T}_{f}}{\partial x_{i}} n_{i} \delta_{w}\right\rangle_{f}=\frac{\left\langle\bar{\Phi} \delta_{w}\right\rangle_{f}}{\left(\rho C_{p}\right)_{f}}$.

When a porous medium is periodic, tortuosity vanishes. This is the case for parallel flows in channels or pipes and tortuosity contribution is zero. Hence, the macroscopic mean temperature equation reads:

$$
\begin{aligned}
\frac{\partial\left\langle\bar{T}_{f}\right\rangle_{f}}{\partial t}+\left\langle\bar{u}_{i}\right\rangle_{f} \frac{\partial\left\langle\bar{T}_{f}\right\rangle_{f}}{\partial x_{i}}= & \frac{\partial}{\partial x_{j}}\left[\left(\alpha_{f}+\alpha_{t \phi}\right) \frac{\partial\left\langle\bar{T}_{f}\right\rangle_{f}}{\partial x_{j}}\right]+\frac{\left\langle\bar{\Phi} \delta_{w}\right\rangle_{f}}{\left(\rho C_{p}\right)_{f}} \\
& +\frac{\partial}{\partial x_{i}}\left(\mathcal{D}_{i j}^{P} \frac{\partial\left\langle\bar{T}_{f}\right\rangle_{f}}{\partial x_{j}}\right)+\frac{\partial}{\partial x_{i}}\left(\mathcal{D}_{i}^{A} \frac{\left\langle\bar{\Phi} \delta_{w}\right\rangle_{f}}{\left(\rho C_{p}\right)_{f}}\right),
\end{aligned}
$$

where $\mathcal{D}_{i j}^{P}$ and $\mathcal{D}_{i}^{A}$ are dispersion tensors. Specific models have been proposed and calibrated for those quantities in [6].

\section{Connecting temperature deviation modeling with algebraic heat exchange models}

In this section, we aim to achieve consistency between the upscaling procedure used to derive the macroscopic temperature equation and wall temperature modeling. Indeed, in order to derive their macroscopic mean temperature model, Drouin et al. [6] used the Carbonell and Whitaker [2] closure relationship, valid for periodic porous media, that tightly relates the temperature deviation to mean temperature gradient and wall heat flux. We analyze here the possibility to connect this closure with wall temperature prediction.

\subsection{Forced convection classical heat exchange models}

In forced convection, the most common way to model heat transfer at a macroscopic scale is to assume that the wall heat flux is proportional to the difference between the wall and bulk temperatures:

$\Phi=h\left(T_{w}-T_{B}\right)$,

where

$T_{B}=\frac{\left\langle u T_{f}\right\rangle_{f}}{\langle u\rangle_{f}}=\left\langle T_{f}\right\rangle_{f}+\frac{\left\langle\delta u \delta T_{f}\right\rangle_{f}}{\langle u\rangle_{f}}$,

and

$h=\frac{N u D_{h}}{\left(\rho C_{p}\right)_{f} \alpha_{f}}$.
In other word, wall temperature is connected with bulk temperature through the heat exchange coefficient and the thermal heat flux according to:

$T_{w}=T_{B}+\frac{\Phi}{h}$.

The Nusselt number $\mathrm{Nu}$ quantifies the ratio between conductive and convective heat transfer. For forced convection flows in ducts, one generally uses correlations relating Nusselt number to Reynolds and Prandtl numbers. Among all correlations (see [10] for a review of available correlations), Colburn [4] or Dittus-Boelter [5] are the most used. They read

$N u=\max \left(N u^{L}, N u^{T}\right)$,

where $N u^{L}$ is a constant that depends on the geometry and

$N u^{T}=A \operatorname{Re}^{m} \operatorname{Pr}^{n}$.

Classical models give satisfactory results for steady established flows with uniform wall heat flux and are very easy to use. However they are not able to accurately predict heat transfer for fast transients or when the wall heat flux is highly non-uniform. For instance, if one injects a temperature burst in an adiabatic pipe, such kind of models will predict that wall temperature is everywhere and always equal to bulk temperature. It is obviously not the case!

\subsection{Algebraic wall temperature model based on a porous media approach}

We aim here to achieve consistency between the upscaling procedure used to derive the macroscopic temperature equation and wall temperature modeling. Indeed, in order to derive their macroscopic mean temperature model, Drouin et al. [6] used the Carbonell and Whitaker [2] closure relationship, valid for periodic porous media:

$\delta \bar{T}_{f}=\bar{T}_{f}-\left\langle\bar{T}_{f}\right\rangle_{f}=\eta_{j} \frac{\partial\left\langle\bar{T}_{f}\right\rangle_{f}}{\partial x_{j}}+\zeta \frac{\left\langle\bar{\Phi} \delta_{w}\right\rangle_{f}}{\left(\rho C_{p}\right)_{f}}$.

In other word, we analyze here the possibility to connect $\left\langle\bar{T}_{f}\right\rangle_{f}$ and Eq. (16), through definition (1), in order to derive a model for the wall temperature. Doing so, we aim to account for dispersion effect upon wall temperature calculation.

In (16), functions $\eta_{j}$ and $\zeta$ are solutions of the following closure problem (see [6])

Inside the REV : $\bar{u}_{i} \frac{\partial \eta_{j}}{\partial x_{i}}-\frac{\partial}{\partial x_{i}}\left[\left(\alpha_{f}+\alpha_{t}\right) \frac{\partial \eta_{j}}{\partial x_{i}}\right]=\frac{\partial \alpha_{t}}{\partial x_{j}}-\delta \bar{u}_{j}$,

$\bar{u}_{i} \frac{\partial \zeta}{\partial x_{i}}-\frac{\partial}{\partial x_{i}}\left[\left(\alpha_{f}+\alpha_{t}\right) \frac{\partial \zeta}{\partial x_{i}}\right]=-1$,

at walls $: \frac{\partial \eta_{j}}{\partial x_{i}} n_{i}=-\delta_{i j} n_{i}$,

$\alpha_{f} \frac{\partial \zeta}{\partial x_{i}} n_{i}=\frac{\bar{\Phi}}{\left\langle\bar{\Phi} \delta_{w}\right\rangle_{f}}$

Using the same approach, we now focus on near wall temperature modeling. Using Eq. (16) one can write

$T_{w}=\frac{\left\langle\bar{T}_{f} \delta_{w}\right\rangle_{f}}{\left\langle\delta_{w}\right\rangle_{f}}=\left\langle\bar{T}_{f}\right\rangle_{f}+\eta_{j_{w}} \frac{\partial\left\langle\bar{T}_{f}\right\rangle_{f}}{\partial x_{j}}+\zeta_{w} \frac{\left\langle\bar{\Phi} \delta_{w}\right\rangle_{f}}{\left(\rho C_{p}\right)_{f}}$.

where

$\eta_{j_{w}}=\frac{\left\langle\eta_{j} \delta_{w}\right\rangle_{f}}{\left\langle\delta_{w}\right\rangle_{f}}, \quad \zeta_{w}=\frac{\left\langle\zeta \delta_{w}\right\rangle_{f}}{\left\langle\delta_{w}\right\rangle_{f}}$. 
To close the wall temperature modeling, $\eta_{j w}$ and $\zeta_{w}$ are needed. Given the form of the closure problem, $\eta_{j w}$ and $\zeta_{w}$ are only known up to a constant (see [6]). This difficulty can be avoided using the idempotence property of the spatial filter $[14,15]$ in Eq. (16):

$\left\langle\delta \bar{T}_{f}\right\rangle_{f}=\left\langle\eta_{j}\right\rangle_{f} \frac{\partial\left\langle\bar{T}_{f}\right\rangle_{f}}{\partial x_{j}}+\langle\zeta\rangle_{f} \frac{\left\langle\bar{\Phi} \delta_{w}\right\rangle_{f}}{\left(\rho C_{p}\right)_{f}}=0$.

A general formulation for the wall temperature is then obtained by subtracting Eq. (23) to Eq. (21)

$T_{w}=\left\langle\bar{T}_{f}\right\rangle_{f}+\Delta \eta_{j} \frac{\partial\left\langle\bar{T}_{f}\right\rangle_{f}}{\partial x_{j}}+\Delta \zeta \frac{\left\langle\bar{\Phi} \delta_{w}\right\rangle_{f}}{\left(\rho C_{p}\right)_{f}}$

where $\Delta \eta_{j}=\eta_{j w}-\left\langle\eta_{j}\right\rangle_{f}$ and $\Delta \zeta=\zeta_{w}-\langle\zeta\rangle_{f}$.

3.2.1. Determination of the model parameters for parallel flows in channels

For parallel flows in channels, Eq. (24) simplifies to

$T_{w}=\left\langle\bar{T}_{f}\right\rangle_{f}+\Delta \eta_{z} \frac{\partial\left\langle\bar{T}_{f}\right\rangle_{f}}{\partial z}+\Delta \zeta \frac{\left\langle\bar{\Phi} \delta_{w}\right\rangle_{f}}{\left(\rho C_{p}\right)_{f}}$.

For laminar flows in plane channels or circular pipes, the closure problem (Eqs. (17)-(20)) can be analytically solved. This calculation allows us to show that $\Delta \eta_{z}$ is identical to the active dispersion coefficient introduced in [6]. The values found for $\Delta \eta_{z}$ and $\Delta \zeta$ are summarized in Table 1.

Note that the closure relationship (25) used with the coefficients given in Table 1 is strictly consistent with the analytical solution of local temperature equation for established laminar flows with constant wall heat flux.

For turbulent flows, analytical expressions cannot be found for $\Delta \eta_{z}$ and $\Delta \zeta$ and they have to be modeled. To this aim, simulations were carried out for a large range of Reynolds numbers and Prandtl numbers. Velocity profiles result from RANS calculations for turbulent regime. To perform the latter RANS simulations, the low-Reynolds number $\bar{k}-\bar{\varepsilon}$ Chien model [3] is used. Since we consider parallel flows, velocity and thermal diffusivity profiles are calculated in 1D. The closure problem (Eqs. (17)-(20)) is discretized and numerically solved in the section of the unit cell.

As for laminar flows, we observe that the model coefficient $\Delta \eta_{z}$ is equal to the active dispersion coefficient introduced in [6]. The model given by Drouin et al. [6] for $\mathcal{D}_{z}^{A}$ is thus used here to model $\Delta \eta_{z}$ (see Section 4 in Drouin et al. [6]). The model coefficient $\Delta \zeta$ is modeled considering that Eq. (25) must be consistent with classical models for established flows with uniform wall heat flux. In this restricted framework, we have

$T_{w}=T_{B}+\frac{\left\langle\bar{\Phi} \delta_{w}\right\rangle_{f}}{\left(\rho C_{p}\right)_{f}} \times \frac{D_{h}^{2}}{4 \alpha_{f} N u}$,

and for such flows, mean temperature equation simplifies to

$\frac{\partial\left\langle\bar{T}_{f}\right\rangle_{f}}{\partial z}=\frac{\left\langle\bar{\Phi} \delta_{w}\right\rangle_{f}}{\left\langle\bar{u}_{z}\right\rangle_{f}\left(\rho C_{p}\right)_{f}}$.

Combining (25)-(27)

$T_{B}+\frac{\left\langle\bar{\Phi} \delta_{w}\right\rangle_{f}}{\left(\rho C_{p}\right)_{f}} \times \frac{D_{h}^{2}}{4 \alpha_{f} N u}=\left\langle\bar{T}_{f}\right\rangle_{f}+\left(\frac{\Delta \eta_{z}}{\left\langle\bar{u}_{z}\right\rangle_{f}}+\Delta \zeta\right) \frac{\left\langle\bar{\Phi} \delta_{w}\right\rangle_{f}}{\left(\rho C_{p}\right)_{f}}$,

Table 1

Values of the model coefficients $\Delta \eta_{z}$ and $\Delta \zeta$ for laminar flows in ducts.

\begin{tabular}{lll}
\hline & Plane channel & Circular pipe \\
\hline$\Delta \eta_{z} / D_{h}$ & $P e / 240$ & $P e / 96$ \\
$\alpha_{f} / D_{h}^{2} \Delta \zeta$ & $1 / 48$ & $1 / 32$ \\
\hline
\end{tabular}

and consequently

$\frac{\Delta \eta_{z}}{\left\langle\bar{u}_{z}\right\rangle_{f}}+\Delta \zeta=\frac{\left(\rho C_{p}\right)_{f}}{\left\langle\bar{\Phi} \delta_{w}\right\rangle_{f}} \times\left(T_{B}-\left\langle\bar{T}_{f}\right\rangle_{f}+\frac{\left\langle\bar{\Phi} \delta_{w}\right\rangle_{f}}{\left(\rho C_{p}\right)_{f}} \times \frac{D_{h}^{2}}{4 \alpha_{f} N u}\right)$,

Using the thermal dispersion model of Drouin et al. [6], Eq. (11) gives

$T_{B}-\left\langle\bar{T}_{f}\right\rangle_{f}=-\left\langle\bar{u}_{z}\right\rangle_{f}^{-1} \mathcal{D}_{z z}^{P} \frac{\partial\left\langle\bar{T}_{f}\right\rangle_{f}}{\partial z}-\left\langle\bar{u}_{z}\right\rangle_{f}^{-1} \mathcal{D}_{z}^{A} \frac{\left\langle\bar{\Phi} \delta_{w}\right\rangle_{f}}{\left(\rho C_{p}\right)_{f}}$,

Eq. (30) is then injected in Eq. (29) and the following relationship can be written

$\Delta \zeta^{*}=\frac{\Delta \zeta \alpha_{f}}{D_{h}^{2}}=\frac{1}{4 N u}-\frac{\mathcal{D}_{z z}^{P}}{\alpha_{f} P e^{2}}-2 \times \frac{\mathcal{D}_{z}^{A}}{D_{h} P e}$

Applied to the cases of plane channel and circular pipe, function (31) has been validated for a broad range of Re number (see Fig. 1). A detailed description of the dispersion coefficients may be found in [6]. The following algebraic model is finally proposed for $T_{w}$ :

$T_{w}=\left\langle\bar{T}_{f}\right\rangle_{f}+\Delta \eta_{z} \frac{\partial\left\langle\bar{T}_{f}\right\rangle_{f}}{\partial z}+\Delta \zeta \frac{\left\langle\bar{\Phi} \delta_{w}\right\rangle_{f}}{\left(\rho C_{p}\right)_{f}}$,

$\Delta \eta_{z}=\mathcal{D}_{z}^{A}$

$\Delta \zeta=\frac{D_{h}^{2}}{\alpha_{f}}\left(\frac{1}{4 N u}-\frac{\mathcal{D}_{z z}^{P}}{\alpha_{f} P e^{2}}-2 \times \frac{\mathcal{D}_{z}^{A}}{D_{h} P e}\right)$.

This model is consistent with porous media model of Drouin et al. [6] and though implicitely accounts for Taylor dispersion. However, since it is based upon the same assumptions than the closure problem, its use must be limited to smooth flow configurations.

\section{Wall temperature transport equation model}

When mean temperature gradients are very stiff or in the presence or highly heterogeneous wall heat fluxes, algebraic model is less valid and accurate. Under those conditions, the scale separation assumption is not verified. Furthermore, wall temperature is mainly controlled by boundary conditions and immediately reacts to variations of the heat flux. Consequently, time and length scales involved in mean temperature and wall temperature might be very different if large temperature variation or stiff heat flux gradients are met. For such flows, a more complex model is needed.

\subsection{General formulation}

To overcome the limitations of algebraic models, a transport equation is derived for $\Delta T=T_{w}-\left\langle\bar{T}_{f}\right\rangle_{f}$ using a two-layer model. Let us consider a parallel flow in a duct of constant section (see Fig. 2). Two distinct regions are defined: a near wall region, denoted $\Omega_{2}$, and a central region $\Omega_{1}$. The frontier of the elementary volume $\Omega$ is $\Sigma$ and $\Sigma_{2,1}$ is the frontier between $\Omega_{1}$ and $\Omega_{2}$. A spatial averaging procedure is defined for each region. It is denoted: $\langle\cdot\rangle_{1,2}$ for regions 1 and 2 respectively. In each region $i$, a mean temperature $T_{i}$, a bulk temperature $T_{B_{i}}$ and a mean velocity $u_{i}$ represent the mean flow variables:

$u_{1,2} \equiv\langle\bar{u}\rangle_{1,2}$

$T_{1,2} \equiv\left\langle\bar{T}_{f}\right\rangle_{1,2}$

$u_{1,2} T_{B_{1,2}} \equiv\left\langle\bar{u} \bar{T}_{f}\right\rangle_{1,2}$.

In such a framework, we have the following relations: 


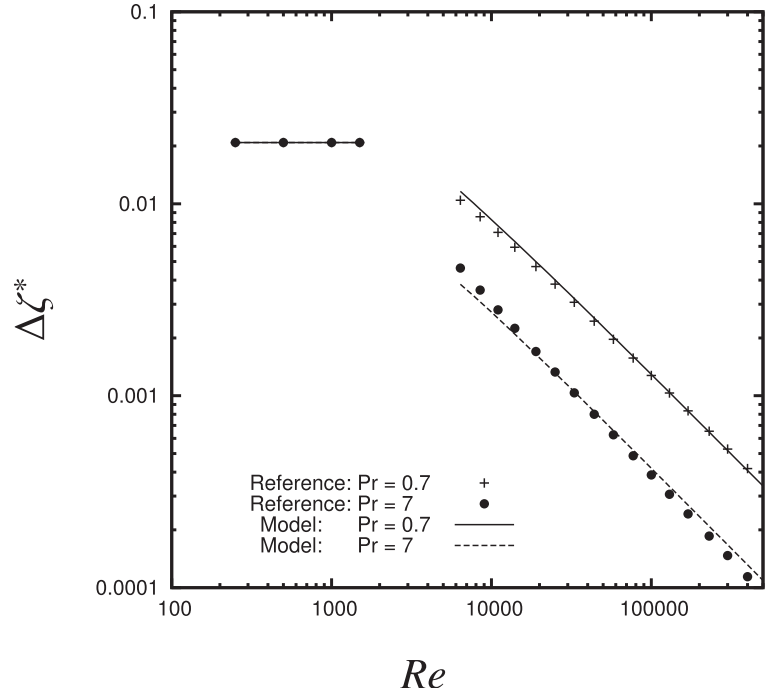

(a) Plane channel

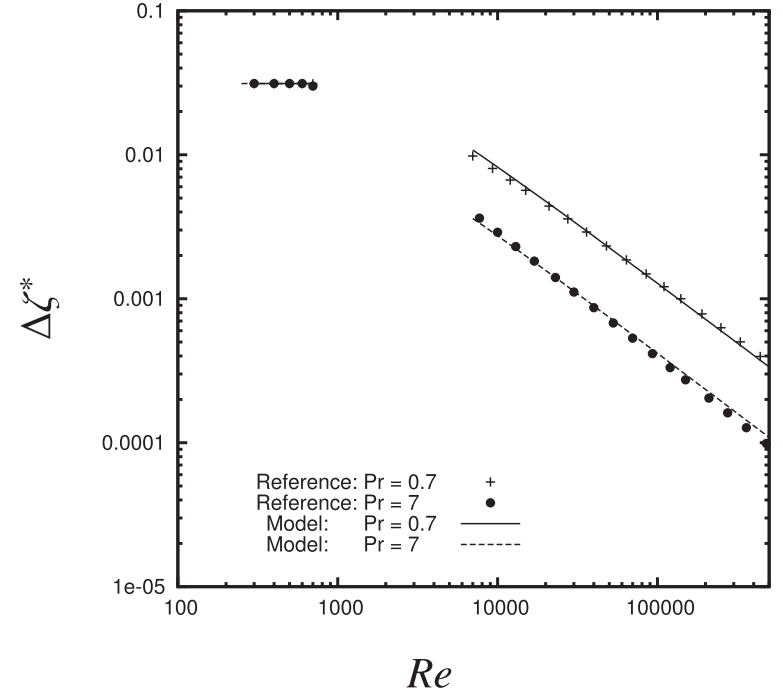

(b) Circular pipe

Fig. 1. Parallel flows in ducts: evolution of $\Delta \zeta^{*}$ as a function of the Reynolds number for several Prandtl numbers. Comparison with numerical reference results.

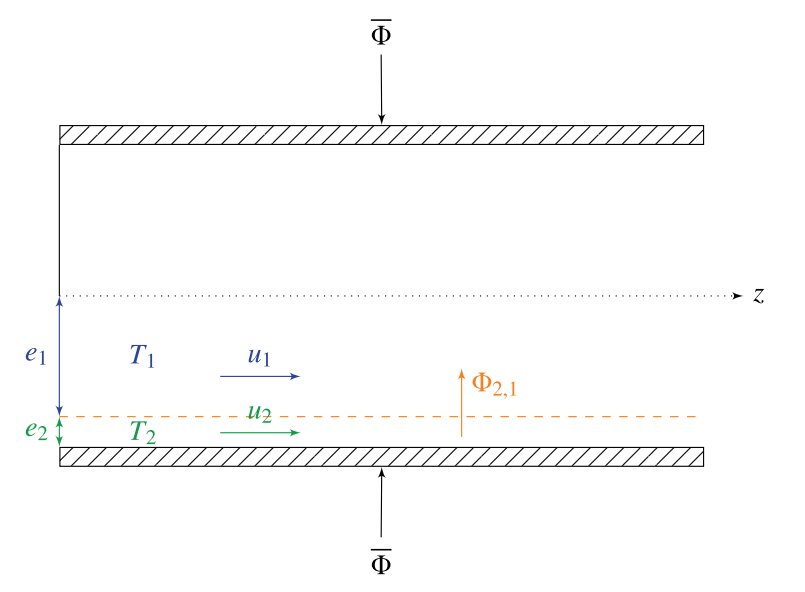

(a) Longitudinal view

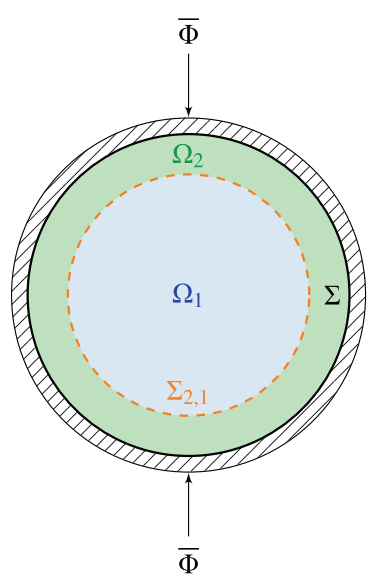

(b) View of a section

Fig. 2. Schematic description of the two-layers decomposition: case of a circular pipe. Solid surface is denoted $\Sigma$ while the surface delimiting both regions is denoted $\Sigma_{2,1}$.

$$
\begin{aligned}
& \Omega_{1}+\Omega_{2}=\Omega \\
& \Omega_{1} T_{1}+\Omega_{2} T_{2}=\Omega\left\langle\bar{T}_{f}\right\rangle_{f} \\
& \Omega_{1} u_{1}+\Omega_{2} u_{2}=\Omega\langle\bar{u}\rangle_{f} \\
& \Omega_{1} u_{1} T_{B_{1}}+\Omega_{2} u_{2} T_{B_{2}}=\Omega\langle\bar{u}\rangle_{f} T_{B}
\end{aligned}
$$

where Eqs. (40) and (41) defines total mass flow rate and total energy flux. Furthermore, let us introduce the following definitions:

$\frac{\Omega_{1}}{\Omega} \equiv \theta$
$\frac{\Omega_{2}}{\Omega} \equiv 1-\theta$.

As a first approximation, we shall assume that turbulent and laminar diffusive contributions along the main flow direction are negligible with respect to advection and dispersion contributions [13]. The wall heat flux is assumed constant over a cross section and reads:

$\left\langle\bar{\Phi} \delta_{w}\right\rangle_{f}=\frac{\Sigma \bar{\Phi}}{\Omega}$.

In order to model heat flux between regions 1 and 2 , we also introduce an internal heat transfer coefficient $h_{2,1}$ :

$\Phi_{2,1}=h_{2,1}\left(T_{2}-T_{1}\right)$

Thanks to definition (39), relation (45) also reads

$\Phi_{2,1}=h_{2,1} \frac{T_{2}-\left\langle\bar{T}_{f}\right\rangle_{f}}{\theta}$.

According to definitions above and thanks to relations (38)-(41), we split the temperature transport equation into transport equations for $T_{1}$ and $T_{2}$ :

$$
\begin{aligned}
& \frac{\partial \theta T_{1}}{\partial t}+\frac{\partial \theta u_{1} T_{B_{1}}}{\partial z}=\frac{\Sigma_{2,1} \Phi_{2,1}}{\Omega\left(\rho C_{p}\right)_{f}}, \\
& \frac{\partial(1-\theta) T_{2}}{\partial t}+\frac{\partial(1-\theta) u_{2} T_{B_{2}}}{\partial z}=\frac{\Sigma \bar{\Phi}}{\Omega\left(\rho C_{p}\right)_{f}}-\frac{\Sigma_{2,1} \Phi_{2,1}}{\Omega\left(\rho C_{p}\right)_{f}} .
\end{aligned}
$$

Adding Eqs. (47) and (48) allows us to recover the macroscopic 1D transport equation for temperature: 
$\frac{\partial\left\langle\bar{T}_{f}\right\rangle_{f}}{\partial t}+\frac{\partial\langle\bar{u}\rangle_{f} T_{B}}{\partial z}=\frac{\Sigma \bar{\Phi}}{\Omega\left(\rho C_{p}\right)_{f}}$

This result garantees that Eqs. (47) and (48) respect the conservation of energy principle. Let us divide Eq. (47) by $\theta$ and Eq. (48) by $1-\theta$ and substract the resulting balance equations. We thus derive the balance equation:

$$
\begin{aligned}
\frac{\partial\left(T_{2}-T_{1}\right)}{\partial t}+\frac{\partial\left(u_{2} T_{B_{2}}-u_{1} T_{B_{1}}\right)}{\partial z}= & \frac{\Sigma \bar{\Phi}}{(1-\theta) \Omega\left(\rho C_{p}\right)_{f}}-\frac{\Sigma_{2,1} \Phi_{2,1}}{\Omega\left(\rho C_{p}\right)_{f}} \\
& \times \frac{1}{\theta(1-\theta)}
\end{aligned}
$$

From now, we shall link each contribution in (50) with $\Delta T=T_{w}-\left\langle\bar{T}_{f}\right\rangle_{f}$. Let us start with time derivative contribution. Thanks to definition (39), we have

$T_{2}-T_{1}=\frac{T_{2}-\left\langle\bar{T}_{f}\right\rangle_{f}}{\theta}$.

Since the wall layer (layer 2) is supposed to be thin, it is assumed that $T_{w}$ and $T_{2}$ evolve simultaneously. In other word, the time necessary to achieve a $T_{2}$ temperature in equilibrium with wall boundary condition is much smaller than that necessary to achieve equilibrium between inner and outer regions of the flow. Under this assumption, $\left(T_{w}-T_{2}\right)$ can be assumed constant in the time derivative in the left hand side of Eq. (50):

$\frac{\partial\left(T_{2}-T_{1}\right)}{\partial t}=\frac{\partial}{\partial t} \frac{\left(T_{w}-\left\langle\bar{T}_{f}\right\rangle_{f}\right)}{\theta} \simeq \frac{1}{\theta} \frac{\partial \Delta T}{\partial t}$.

Using (41), we show that

$$
\begin{aligned}
T_{B_{2}} u_{2}-T_{B_{1}} u_{1}= & \frac{T_{B_{2}} u_{2}-T_{B}\langle\bar{u}\rangle_{f}}{\theta}=\frac{T_{B_{2}} u_{2}-\left\langle\bar{T}_{f}\right\rangle_{f}\langle\bar{u}\rangle_{f}-\left\langle\delta \bar{T}_{f} \delta \bar{u}\right\rangle_{f}}{\theta} \\
= & \frac{1}{\theta}\left[\left(T_{B_{2}}-\left\langle\bar{T}_{f}\right\rangle_{f}\right) u_{2}-\left\langle\bar{T}_{f}\right\rangle_{f}\left(\langle\bar{u}\rangle_{f}-u_{2}\right)\right. \\
& \left.-\left\langle\delta \bar{T}_{f} \delta \bar{u}\right\rangle_{f}\right] .
\end{aligned}
$$

Furthermore, since layer 2 (wall layer) is assumed thin, it is physically acceptable to link $T_{B_{2}}$ and $T_{w}$ by a wall heat exchange coefficient

$\bar{\Phi}=h_{w}\left(T_{w}-T_{B_{2}}\right)$.

Hence, advection contribution in (50) may read:

$$
\begin{aligned}
\frac{\partial\left(T_{B_{2}} u_{2}-T_{B_{1}} u_{1}\right)}{\partial z}= & \frac{1}{\theta} \frac{\partial}{\partial z}\left[\left(T_{B_{2}}-\left\langle\bar{T}_{f}\right\rangle_{f}\right) u_{2}-\left\langle\bar{T}_{f}\right\rangle_{f}\left(\langle\bar{u}\rangle_{f}-u_{2}\right)\right. \\
& \left.-\left\langle\delta \bar{T}_{f} \delta \bar{u}\right\rangle_{f}\right] \\
= & \frac{u_{2}}{\theta} \frac{\partial T_{w}-\left\langle\bar{T}_{f}\right\rangle_{f}}{\partial z}-\frac{u_{2}}{h_{w} \theta} \frac{\partial \bar{\Phi}}{\partial z}-\frac{\langle\bar{u}\rangle_{f}-u_{2}}{\theta} \\
& \times \frac{\partial\left\langle\bar{T}_{f}\right\rangle_{f}}{\partial z}-\frac{1}{\theta} \frac{\partial\left\langle\delta \bar{T}_{f} \delta \bar{u}\right\rangle_{f}}{\partial z} .
\end{aligned}
$$

Velocities are constant along $z$. Taking into account relations (53) and (46), we express the contribution involving the heat flux between regions 1 and 2 in (50) as

$$
\begin{aligned}
\frac{\Sigma_{2,1} \Phi_{2,1}}{\Omega\left(\rho C_{p}\right)_{f}} \times \frac{1}{\theta(1-\theta)} & =\frac{\Sigma_{2,1} h_{2,1}}{\Omega\left(\rho C_{p}\right)_{f}} \times \frac{T_{2}-\left\langle\bar{T}_{f}\right\rangle_{f}}{\theta} \times \frac{1}{\theta^{2}(1-\theta)} \\
& \simeq \frac{\Sigma_{2,1} h_{2,1}}{\Omega\left(\rho C_{p}\right)_{f}} \times\left(\Delta T-\frac{\bar{\Phi}}{h_{w}}\right) \times \frac{1}{\theta(1-\theta)} .
\end{aligned}
$$

Finally, we introduce (51), (54) and (55) in Eq. (50) to get after some algebra

$$
\begin{aligned}
\frac{\partial \Delta T}{\partial t}+u_{2} \frac{\partial \Delta T}{\partial z}= & \frac{\bar{\Phi}}{\Omega\left(\rho C_{p}\right)_{f}} \times \frac{1}{1-\theta}\left(\theta \Sigma+\frac{\Sigma_{2,1} h_{2,1}}{\theta h_{w}}\right) \\
& -\frac{\Sigma_{2,1} h_{2,1}}{\Omega \theta(1-\theta)\left(\rho C_{p}\right)_{f}} \Delta T+\left(\langle\bar{u}\rangle_{f}-u_{2}\right) \frac{\partial\left\langle\bar{T}_{f}\right\rangle_{f}}{\partial z} \\
& +\frac{\partial\left\langle\delta \bar{T}_{f} \delta \bar{u}\right\rangle_{f}}{\partial z}+\frac{u_{2}}{h_{w}} \frac{\partial \bar{\Phi}}{\partial z} .
\end{aligned}
$$

For flows with smooth mean temperature gradient and uniform wall heat fluxes, the scale separation assumption is valid. In other words, $\Delta T$ must achieve the asymptotic limit given by the algebraic model (32):

$\Delta T=\Delta \eta_{z} \frac{\partial\left\langle\bar{T}_{f}\right\rangle_{f}}{\partial z}+\Delta \zeta \frac{\left\langle\bar{\Phi} \delta_{w}\right\rangle_{f}}{\left(\rho C_{p}\right)_{f}}$,

and the transport terms in Eq. (56) can be neglected. Under these restraining assumptions, Eq. (56), reads

$$
\begin{aligned}
\frac{\Sigma_{2,1} h_{2,1}}{\Omega \theta(1-\theta)\left(\rho C_{p}\right)_{f}} \Delta T= & \left(\langle\bar{u}\rangle_{f}-u_{2}\right) \frac{\partial\left\langle\bar{T}_{f}\right\rangle_{f}}{\partial z}+\frac{\bar{\Phi}}{\Omega\left(\rho C_{p}\right)_{f}} \\
& \times \frac{1}{1-\theta}\left(\theta \Sigma+\frac{\Sigma_{2,1} h_{2,1}}{\theta h_{w}}\right)
\end{aligned}
$$

and must be consistent with algebraic model (57). By identification, comparison between Eqs. (58) and (57) leads to

$\Delta \eta_{z}=\frac{\theta(1-\theta) \Omega\left(\rho C_{p}\right)_{f}}{\Sigma_{2,1} h_{2,1}} \times\left(\langle u\rangle_{f}-u_{2}\right)$,

$\Delta \zeta=\frac{\Omega\left(\rho C_{p}\right)_{f}}{\Sigma_{2,1} h_{2,1}} \times\left(\theta^{2}+\frac{\Sigma_{2,1} h_{2,1}}{\Sigma h_{w}}\right)$

Coefficients of the first and second terms of the right hand side of Eq. (56) still need to be determined. Using Eqs. (59) and (60), we show that

$\frac{\Sigma_{2,1} h_{2,1}}{\Omega\left(\rho C_{p}\right)_{f} \theta(1-\theta)} \Delta T=\frac{\langle\bar{u}\rangle_{f}-u_{2}}{\Delta \eta_{z}} \Delta T$,

and

$\frac{\bar{\Phi}}{\Omega\left(\rho C_{p}\right)_{f}} \times \frac{1}{1-\theta}\left(\theta \Sigma+\frac{\Sigma_{2,1} h_{2,1}}{\theta h_{w}}\right)=\left(\langle\bar{u}\rangle_{f}-u_{2}\right) \frac{\Delta \zeta}{\Delta \eta_{z}} \frac{\Sigma \bar{\Phi}}{\Omega\left(\rho C_{p}\right)_{f}}$.

Using Eqs. (59)-(62), Eq. (56) finally reads

$$
\begin{aligned}
\frac{\partial \Delta T}{\partial t}+u_{2} \frac{\partial \Delta T}{\partial z}= & \left(\langle\bar{u}\rangle_{f}-u_{2}\right) \frac{\Delta \zeta}{\Delta \eta_{z}} \frac{\Sigma \bar{\Phi}}{\Omega\left(\rho C_{p}\right)_{f}}-\frac{\langle\bar{u}\rangle_{f}-u_{2}}{\Delta \eta_{z}} \Delta T \\
& +\left(\langle\bar{u}\rangle_{f}-u_{2}\right) \frac{\partial\left\langle\bar{T}_{f}\right\rangle_{f}}{\partial z}+\frac{\partial\left\langle\delta \bar{T}_{f} \delta \bar{u}\right\rangle_{f}}{\partial z}+\frac{u_{2}}{h_{w}} \frac{\partial \bar{\Phi}}{\partial z},
\end{aligned}
$$

where $\Omega_{2}$ (i.e. $e_{2}$ ), $u_{2}$ and $h_{w}$ have to be evaluated.

\subsection{Determination of the model parameters}

The thickness of the near-wall layer can be seen as a characteristic length associated with heat exchange. Thus, we classically propose

$e_{2} \propto \frac{D_{h}}{N u}$

\subsubsection{Laminar flows}

For laminar flows, the thickness of the near-wall layer reads

$e_{2}=\frac{D_{h}}{N u_{f}}$.

Since $\Delta T$ links wall temperature to spatially averaged temperature, Nusselt number $N u_{f}$, for laminar flows, is slightly different from 
classical Nusselt number that links wall to bulk temperatures. For laminar flows in ducts, velocity and temperature profiles can be determined analytically, at least for simple geometries. We find for flows in plane channels: $N u_{f}=10$ and for circular pipes: $N u_{f}=6$. Volume fraction $1-\theta$ and bulk velocity $u_{2}$ of wall region are given by integrating velocity profiles:

$\frac{\Omega_{2}}{\Omega}=1-\theta=4 \frac{e_{2}}{D_{h}}$ and $u_{2}=6\langle\bar{u}\rangle_{f} \frac{e_{2}}{D_{h}}\left(1-\frac{4}{3} \frac{e_{2}}{D_{h}}\right)$ in plane channels,

$\frac{\Omega_{2}}{\Omega}=1-\theta=4 \frac{e_{2}}{D_{h}}\left(1-\frac{e_{2}}{D_{h}}\right)$ and $u_{2}=4\langle\bar{u}\rangle_{f} \frac{e_{2}}{D_{h}}\left(1-\frac{e_{2}}{D_{h}}\right)$ in circular pipes.

The wall layer heat exchange coefficient, introduced in (53), links wall temperature to bulk temperature of wall layer:

$h_{w}=\frac{\bar{\Phi}}{T_{w}-T_{B_{2}}}$.

In order to obtain its expression, we consider that:

$T_{B_{2}}=\frac{\langle T u\rangle_{2}}{u_{2}}=T_{w}+\frac{\left\langle\left(T-T_{w}\right) u\right\rangle_{2}}{u_{2}}$.

Then, we use velocity and temperature profiles for steady and fully developed laminar flows and we integrate those profiles other cross sections. Those profiles reads for plane channels:

$$
\begin{aligned}
& u(y)=\frac{3\langle\bar{u}\rangle_{f}}{2}\left[1-\left(\frac{y}{e}\right)^{2}\right], \\
& T(y)-T_{w}=\frac{3\langle\bar{u}\rangle_{f} e^{2}}{2 \alpha_{f}} \frac{\partial T_{B}}{\partial z}\left[\frac{1}{2}\left(\frac{y}{e}\right)^{2}-\frac{1}{12}\left(\frac{y}{e}\right)^{4}-\frac{5}{12}\right]
\end{aligned}
$$

and for circular pipes:

$$
\begin{aligned}
& u(r)=2\langle\bar{u}\rangle_{f}\left[1-\left(\frac{r}{R}\right)^{2}\right], \\
& T(r)-T_{w}=\frac{\langle\bar{u}\rangle_{f} R^{2}}{2 \alpha_{f}} \frac{\partial T_{B}}{\partial z}\left[\left(\frac{r}{R}\right)^{2} \times\left(1-\frac{1}{4}\left(\frac{r}{R}\right)^{2}\right)-\frac{3}{4}\right] .
\end{aligned}
$$

In Eq. (67), $y$ is the transverse coordinate with the origin $y=0$ located at the symmetry plane of the channel and $e=D_{h} / 4$ is half the gap of the channel. In Eq. (68), $r$ is the radial coordinate with the origin $r=0$ at the center of the pipe and $R=D_{h} / 2$ is the radius of the pipe. We also introduce the non dimensional thickness of the central region denoted $\epsilon$. For plane channels, we use $\epsilon=e_{1} /$ $e=\left(e_{2}-e\right) / e$ and for circular pipes, we use $\epsilon=e_{1} / R=\left(R-e_{2}\right) / R$. For flows in plane channel, we find after some algebra:

$$
h_{w}=\frac{\bar{\Phi}}{T_{w}-T_{B_{2}}}=\frac{140}{17} \frac{\lambda_{f}}{D_{h}} \times \frac{(1-\epsilon)\left[1-\frac{\epsilon}{2}(1+\epsilon)\right]}{1+\frac{105}{272}\left(\frac{\epsilon^{7}}{7}-\frac{7 \epsilon^{5}}{5}+\frac{11 \epsilon^{3}}{3}-5 \epsilon\right)} .
$$

Please note that at the limit where $\epsilon \rightarrow 0$, one recovers the classical Nusselt number for laminar flows in plane channels: $N u=140 / 17$. For flows in circular pipes, we find after some algebra:

$h_{w}=\frac{\bar{\Phi}}{T_{w}-T_{B_{2}}}=\frac{48 \lambda_{f}}{D_{h}} \times \frac{\left(1-\epsilon^{2}\right)^{2}}{11+\epsilon^{2}\left(3 \epsilon^{6}-20 \epsilon^{4}+42 \epsilon^{2}-36\right)}$.

Please also note that at the limit where $\epsilon \rightarrow 0$, one recovers the classical Nusselt number for laminar flows in circular pipes: $N u=48 / 11$.

\subsubsection{Turbulent flows}

In order to close the proposed model, we choose to connect $u_{2}$ and $e_{2}$ to characteristic velocity $\left(u_{\tau}\right)$ and length scale $\left(y^{*}\right)$ of classical turbulent boundary layer theory. Please be careful that, instead of Section 4.2.1 where the origin of the coordinate $y$ is the center of the channel (circular pipe respectively), in this section $y$ stands for the distance from wall. For established turbulent flows in pipes, Nusselt number can be approached by a classical model

$N u=A \operatorname{Re}^{m} \operatorname{Pr}^{n}$.

Friction velocity is linked with friction factor and bulk velocity through:

$u_{\tau}=\langle\bar{u}\rangle \sqrt{\frac{f_{w}}{8}}$

and wall unit is defined by: $y^{*} \equiv v_{f} / u_{\tau}$. In order to predict friction factor, we shall use Blasius type of correlations:

$f_{w}=a R e^{-b}$.

Finally, we introduce:

$e_{2}^{+} \equiv \frac{e_{2}}{y^{*}}$.

Expressions (71) and (73) are injected in (64) to obtained

$e_{2}^{+}=\frac{u_{\tau}}{v_{f}} \times \frac{D_{h}}{N u}=\frac{\left\langle\bar{u}_{z}\right\rangle_{f} \sqrt{f_{w}} D_{h}}{v_{f} \sqrt{8} A R e^{m} \operatorname{Pr}^{n}}$.

After some algebra, we find

$e_{2}^{+}=\frac{\sqrt{a}}{A \sqrt{8}} \frac{\operatorname{Re}^{1-b / 2-m}}{\operatorname{Pr}^{n}}$.

For flows in smooth ducts, $b \in[0.19,0.25], m \in[0.8,0.9]$ so that $1-b / 2-m \simeq 0$. Hence, one can use the following approximation

$e_{2}^{+}=\frac{C_{\beta}}{\operatorname{Pr}^{n}} \quad$ where $\quad C_{\beta} \equiv \frac{\sqrt{a}}{A \sqrt{8}}$

and $n \in[1 / 3 ; 1 / 2]$. Classical values for $a$ range within $a \in[0.16,0.316]$ while $A$ ranges within $A \in[0.009,0.024]$. Hence, plausible values for $C_{\beta}$ might be found in the range $C_{\beta} \in[6,22]$. Turbulent boundary layer shows that four regions have to be considered

Viscous sub layer : $0 \leqslant y^{+}<5$,

buffer region : $5 \leqslant y^{+} \leqslant 30$,

logarithmic region : $30<y^{+} \lesssim 1000$,

bulk region : $1000 \lesssim y^{+}$.

In other words, for classical values of the Prandtl number (i.e. $\mathrm{Pr}$ close to one), $e_{2}$ is placed within the so called buffer region of the boundary layer.

We now have to connect $e_{2}$ with its characteristic velocity $u_{2}$. To this aim, we use the following simplified velocity profile

$u^{+}=\frac{u}{u_{\tau}}= \begin{cases}y^{+} & \text {if } y^{+} \leqslant y_{t}^{+}, \\ \frac{1}{\kappa} \ln \left[1+\kappa\left(y^{+}-y_{t}^{+}\right)\right]+y_{t}^{+} & \text {if } y^{+}>y_{t}^{+},\end{cases}$

where $\kappa=0.41$ and $y_{t}^{+}=7.8$. In Eq. (77), $y_{t}^{+}$does not denote the upper limit of the viscous sub-layer but is the connection point

Table 2

Summary of the model parameters for laminar and turbulent flows in ducts.

\begin{tabular}{lllll}
\hline & $\Omega_{2} / \Omega$ & $u_{2}$ (laminar) & $u_{2}$ (turbulent) & $h_{w}$ (turbulent) \\
\hline Plane channel & $4 \frac{e_{2}}{D_{h}}$ & $6\left\langle\bar{u}_{z}\right\rangle_{f} \frac{e_{2}}{D_{h}}\left(1-\frac{4}{3} \frac{e_{2}}{D_{h}}\right)$ & Eq. (79) & Eq. (69) \\
Circular pipe & $4 \frac{e_{2}}{D_{h}}\left(1-\frac{e_{2}}{D_{h}}\right)$ & $4\left\langle\bar{u}_{z}\right\rangle_{f} \frac{e_{2}}{D_{h}}\left(1-\frac{e_{2}}{D_{h}}\right)$ & Eq. (79) & Eq. (70) \\
\hline
\end{tabular}




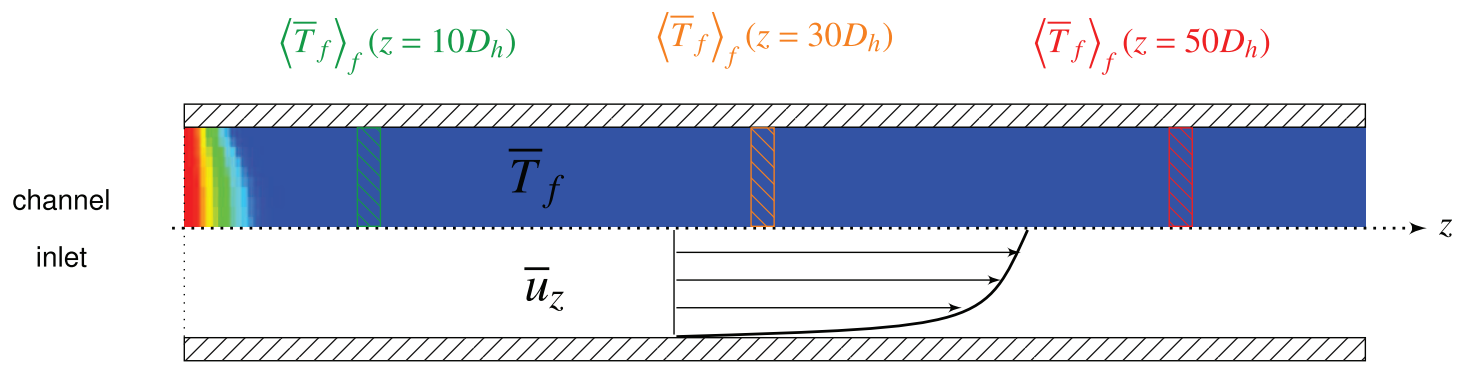

(a) $t_{0}$

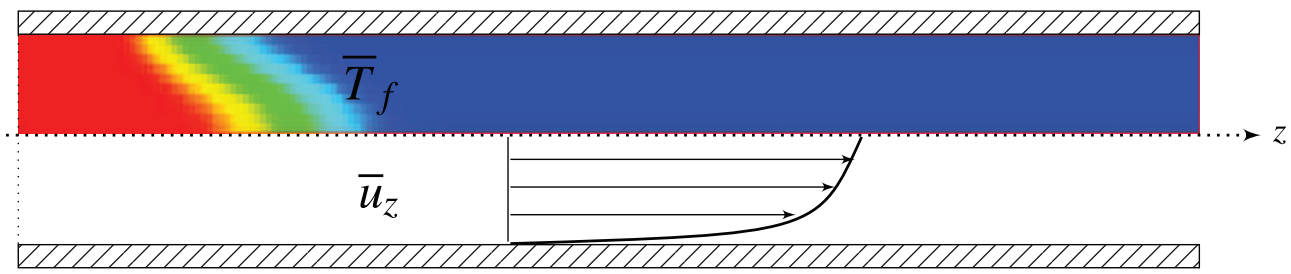

(b) $t_{1}$

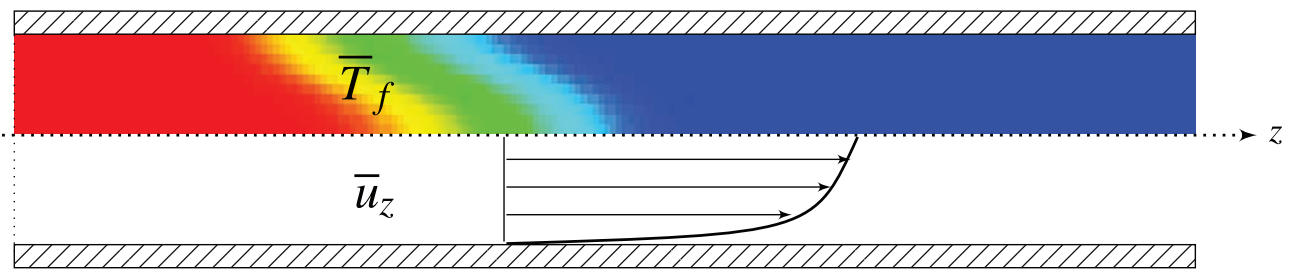

(c) $t_{2}$

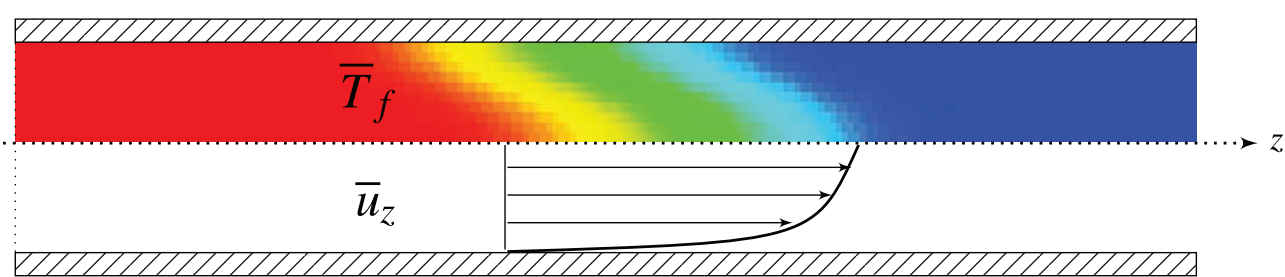

(d) $t_{3}$

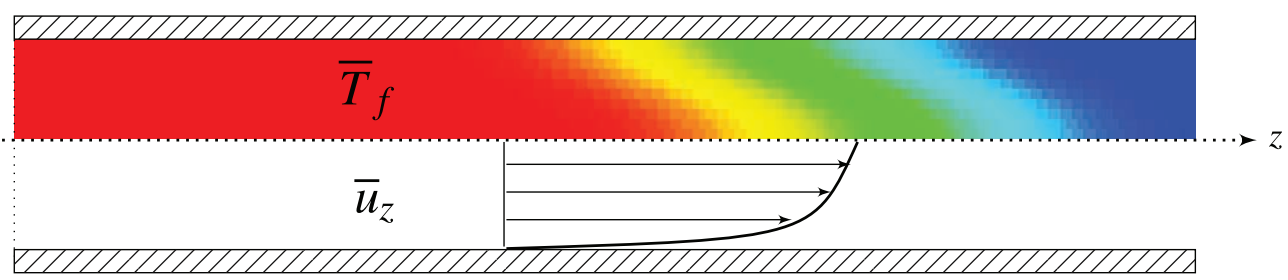

(e) $t_{4}$

Fig. 3. Adiabatic test case: spreading of a temperature jump along the time under the effect of Taylor dispersion.

Table 3

Validation test-cases: adiabatic dispersive flows. Acronym "CP" stands for plane channel and "Tu" for circular pipes.

\begin{tabular}{llc}
\hline & $R e$ & $\operatorname{Pr}$ \\
\hline$C P_{1}$ & 175 & 1.48 \\
$C P_{2}$ & $1.5 \times 10^{4}$ & 0.74 \\
$T u_{1}$ & 175 & 1.48 \\
$T u_{2}$ & $1.5 \times 10^{4}$ & 0.74 \\
\hline
\end{tabular}

between both regions of the simplified profile. The value $y_{t}^{+}=7.8$, placed within the buffer region, allows to approximate the classical Reichardt velocity profile [16]. The wall layer thickness is small with respect to the hydraulic diameter. Consequently, concentricity effect in other geometries than plane channel are negligible. The averaged velocity in the near-wall layer $u_{2}$ is then given by:

$\frac{u_{2}}{u_{\tau}}=\frac{1}{e_{2}} \int_{0}^{e_{2}} u^{+}\left(y^{+}\right) d y^{+}$. 


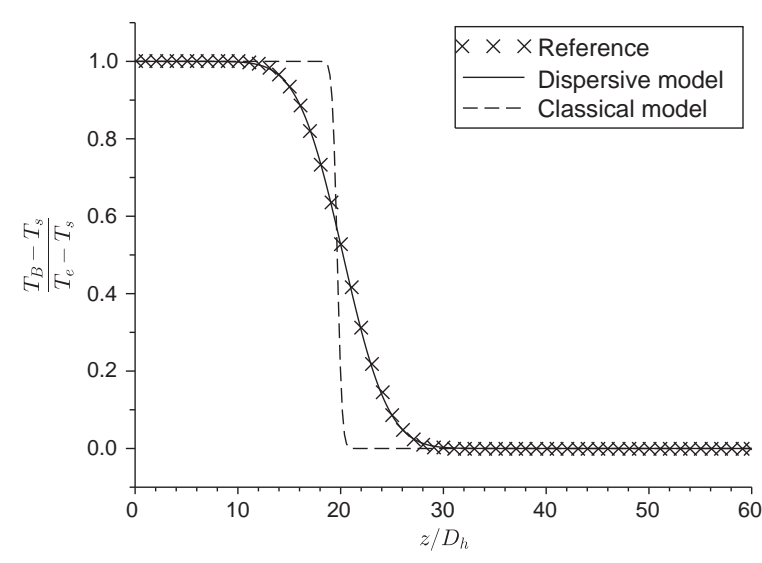

Fig. 4. Temperature jump in a laminar adiabatic flows in a plane channel, $R e=175$ $P r=1.48$ : comparison between classical (Eq. (86)), dispersive (Eq. (30)) and reference results coming from spatially averaged CFD fine scale simulations. Temperature profiles are scaled with inlet $\left(T_{e}\right)$ and outlet $\left(T_{s}\right)$ temperature values.

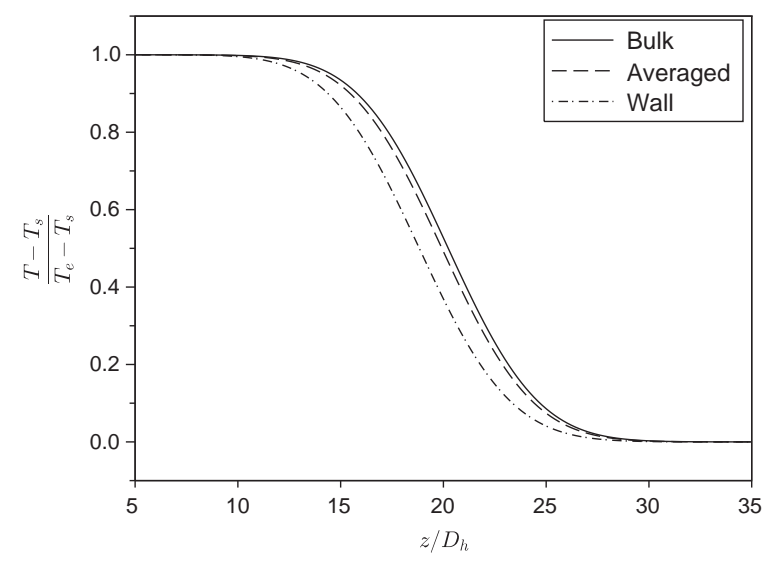

Fig. 5. Temperature jump in a laminar adiabatic flows in a plane channel, $R e=175$ $\operatorname{Pr}=1.48$ : comparison between bulk $\left(T_{B}\right)$, averaged $\left(\left\langle\bar{T}_{f}\right\rangle_{f}\right)$ and wall $\left(T_{w}\right)$ temperature profiles provided by our macroscale model. On can see the shift between bulk temperature increase and wall temperature increase. Temperature profiles are scaled with inlet $\left(T_{e}\right)$ and outlet $\left(T_{s}\right)$ temperature values.

With (77), this leads to

$\frac{u_{2}}{u_{\tau}}= \begin{cases}\frac{e_{2}^{+}}{2} & \text { if } e_{2}^{+} \leqslant y_{t}^{+}, \\ \frac{y_{t}^{+2}}{2 e_{2}^{+}}+\frac{1}{e_{2}^{+}}\left[\left(\frac{1}{\kappa}+e_{2}^{+}-y_{t}^{+}\right) \ln \left(1+\kappa\left(e_{2}^{+}-y_{t}^{+}\right)\right)+\right. & \\ \left.\left(y_{t}^{+}-1\right)\left(e_{2}^{+}-y_{t}^{+}\right)\right] & \text {if } e_{2}^{+}>y_{t}^{+} .\end{cases}$

Finally, we have to establish $h_{w}$ the heat exchange coefficient that links the wall heat flux to $T_{w}-T_{B_{2}}$. In order to obtain its expression, we once again use the following relationship:

$T_{B_{2}}=\frac{\langle T u\rangle_{2}}{u_{2}}=T_{w}+\frac{\left\langle\left(T-T_{w}\right) u\right\rangle_{2}}{u_{2}}$.

For turbulent boundary layers, it is usual to introduce the nondimensional form of temperature profile:

$T^{+}=\frac{T_{w}-T}{T_{\tau}} \quad$ with $\quad T_{\tau}=\frac{\bar{\Phi}}{\rho C_{p} u_{\tau}}$

where, in wall vicinity, temperature profile is given by:

$T^{+}=\operatorname{Pr} \times y^{+}$.

According to those definitions, Eq. (80) may also read
$T_{W}-T_{B_{2}}=T_{\tau} \frac{u_{\tau}}{u_{2}} \frac{\left\langle T^{+} u^{+}\right\rangle_{2}}{u_{2}}=T_{\tau} \frac{u_{\tau}}{u_{2}} \times \frac{y^{*}}{e_{2}} \int_{0}^{e_{2}^{+}} T^{+} u^{+} d y^{+}$.

As a first approximation, and for the sake of simplicity, we shall assume here that velocity profile is linear. After some algebra, we obtain that

$T_{W}-T_{B_{2}}=\frac{2 e_{2}}{3 \lambda_{f}} \times \bar{\Phi} \quad$ or, in other words, that $h_{w}=\frac{3 \lambda_{f}}{2 e_{2}}$.

Finally the wall to mean gap temperature model reads

$$
\begin{aligned}
\frac{\partial \Delta T}{\partial t}+u_{2} \frac{\partial \Delta T}{\partial z}= & \left(\langle\bar{u}\rangle_{f}-u_{2}\right) \frac{\Delta \zeta}{\Delta \eta_{z}} \frac{\Sigma \bar{\Phi}}{\Omega\left(\rho C_{p}\right)_{f}}-\frac{\langle\bar{u}\rangle_{f}-u_{2}}{\Delta \eta_{z}} \Delta T \\
& +\left(\langle\bar{u}\rangle_{f}-u_{2}\right) \frac{\partial\left\langle\bar{T}_{f}\right\rangle_{f}}{\partial z}+\frac{\partial\left\langle\delta \bar{T}_{f} \delta \bar{u}\right\rangle_{f}}{\partial z}+\frac{u_{2}}{h_{w}} \frac{\partial \bar{\Phi}}{\partial z},
\end{aligned}
$$

where the thickness of the near-wall layer is

$e_{2}= \begin{cases}\frac{D_{h}}{N u_{f}} & \text { for laminar flows, } \\ \frac{y^{*} C_{\beta}}{P r^{n}} & \text { for turbulent flows, }\end{cases}$

which leads to the values of $\Omega_{2} / \Omega$ and $u_{2}$ summarized in Table 2 .

Models for $\Delta \zeta$ and $\Delta \eta_{z}$ are summarized in Table 1 for laminar flows and in Eqs. (33) and (34) for turbulent flows. For parameter $n$, we choose: $n=0.4$, consistently with Dittus-Boelter heat exchange model. For parameter $C_{\beta}$, we aim to guarantee that $e_{2}$ is placed within the buffer layer and that its value is consistent with Mac Adams friction factor and Dittus-Boelter heat exchange model. Value $C_{\beta}=15.5$ if consistent with those assumptions. Sensibility analysis has been performed within the range $n \in[1 / 3,1 / 2]$ and $C_{\beta} \in[5,20]$ (see [7]). Its appears that wall temperature weakly depends upon those both constants.

\section{Results and discussion}

In order to illustrate the potentialities of the transport equation temperature model presented in Section 4, solutions of Eq. (84) are compared, on one hand, with reference results coming from finescale simulations and, on the other hand, with results of a classical model. Fine scale simulation results are spatially averaged over the cross section of the pipes and channels in order to achieve 1D profiles along the main flow direction. In industrial codes used to predict heat exchange in nuclear reactor cores or heat exchangers $[1,19]$, thermal dispersion is usually neglected and, consequently, it is implicitly assumed that $T_{B}=\left\langle\bar{T}_{f}\right\rangle_{f}$. Hence, bulk temperature calculated with such codes simply reads

$\frac{\partial T_{B}}{\partial t}+\frac{\partial\langle\bar{u}\rangle_{f} T_{B}}{\partial z}=\frac{\Sigma \bar{\Phi}}{\Omega\left(\rho C_{p}\right)_{f}}+\frac{\partial}{\partial z}\left[\left(\alpha_{f}+\alpha_{t \phi}\right) \frac{\partial T_{B}}{\partial z}\right]$

and wall temperature is given by

$T_{w}=T_{B}+\frac{\bar{\Phi}}{h}$.

In fine scale simulations, the Nusselt number is a result. It is calculated thanks to velocity and temperature profiles within the boundary layer. In macroscopic scale models, the Nusselt number value is given by the model. In order to perform fair comparisons, we have used the same model for the Nusselt number model for both classical heat exchange model and transport equation model. This model matches the results obtain with the low-Reynolds $\bar{k}-\bar{\varepsilon}$ model of Chien [3] for uniformly heated, established flows. This Nusselt number is fairly well approached by

$N u=0.011 \operatorname{Re}^{0.87} \operatorname{Pr}^{0.5}$. 


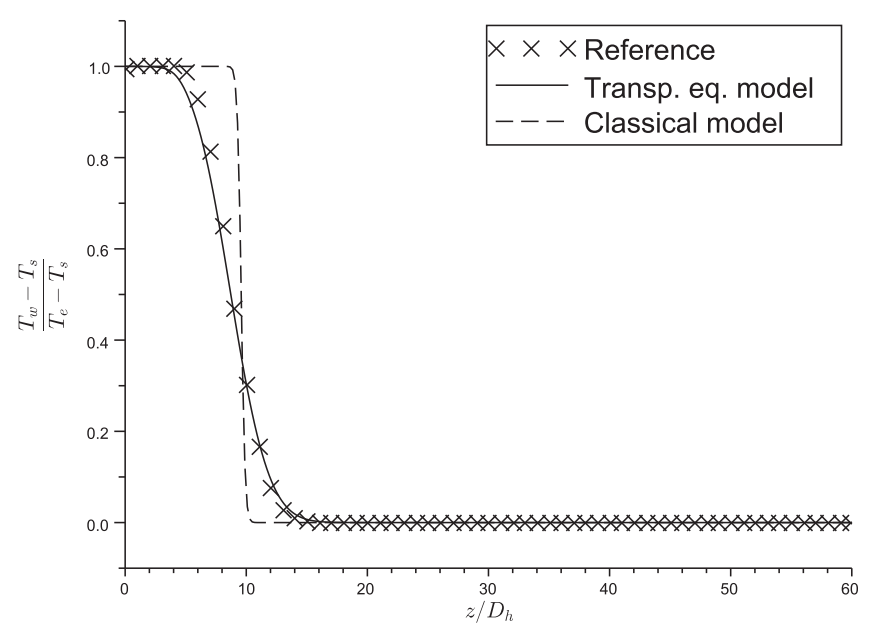

(a) Plane channel $t_{1}=10 D_{h} /\left\langle\bar{u}_{z}\right\rangle_{f}$

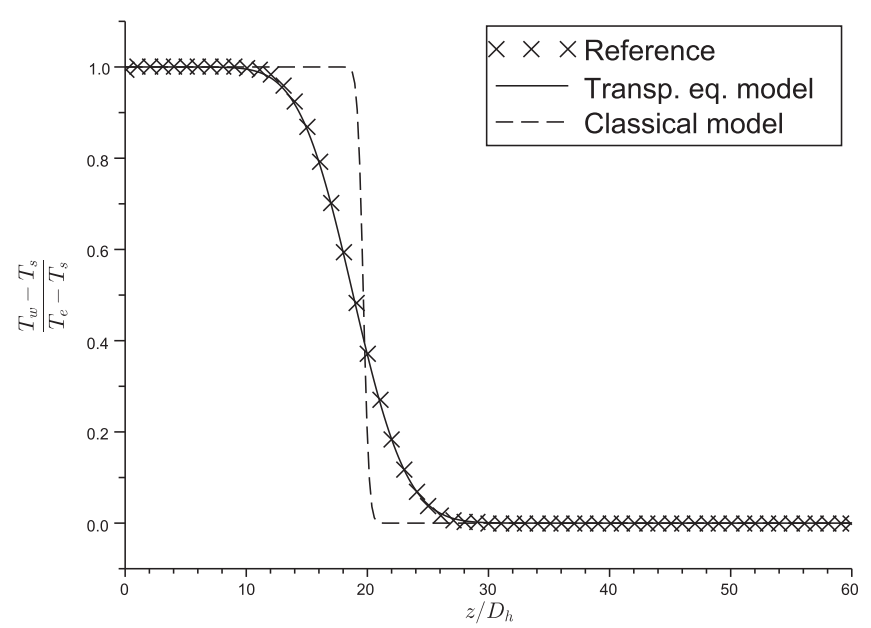

(c) Plane channel $t_{2}=20 D_{h} /\left\langle\bar{u}_{z}\right\rangle_{f}$

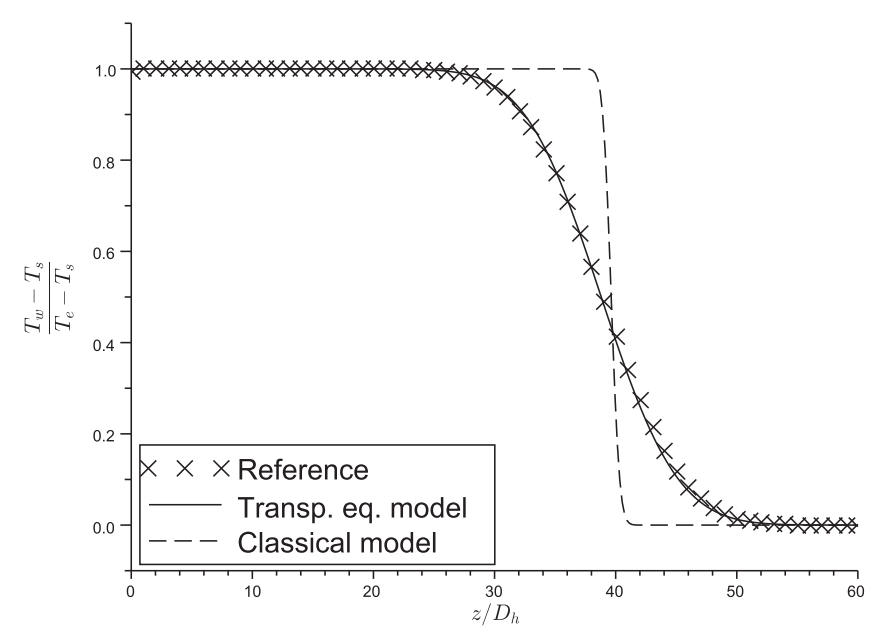

(e) Plane channel $t_{3}=40 D_{h} /\left\langle\bar{u}_{z}\right\rangle_{f}$

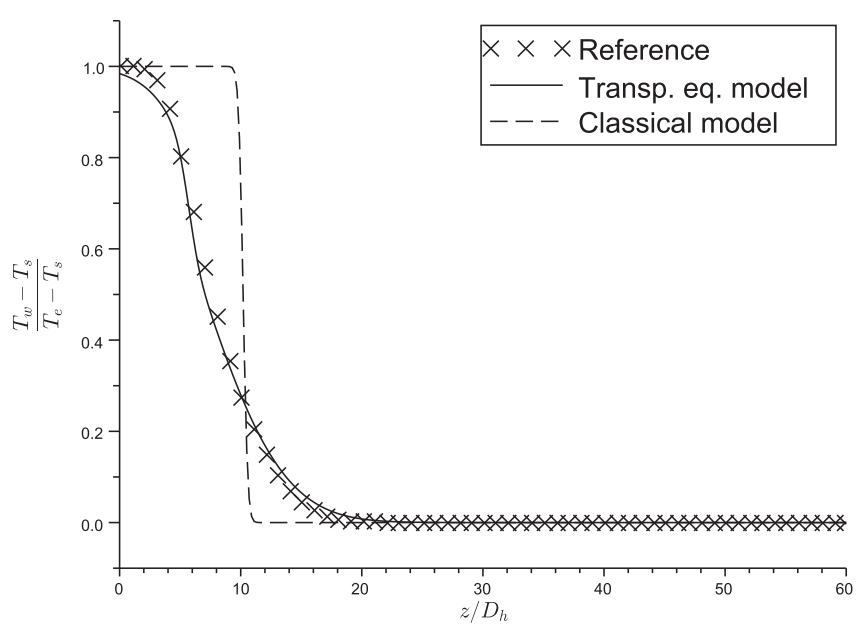

(b) Circular pipe $t_{1}=10 D_{h} /\left\langle\bar{u}_{z}\right\rangle_{f}$

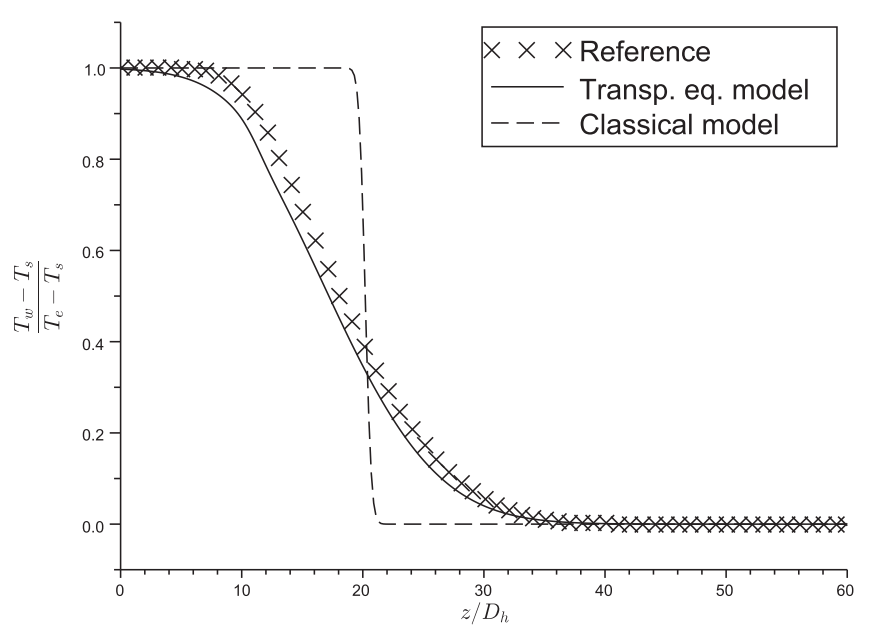

(d) Circular pipe $t_{2}=20 D_{h} /\left\langle\bar{u}_{z}\right\rangle_{f}$

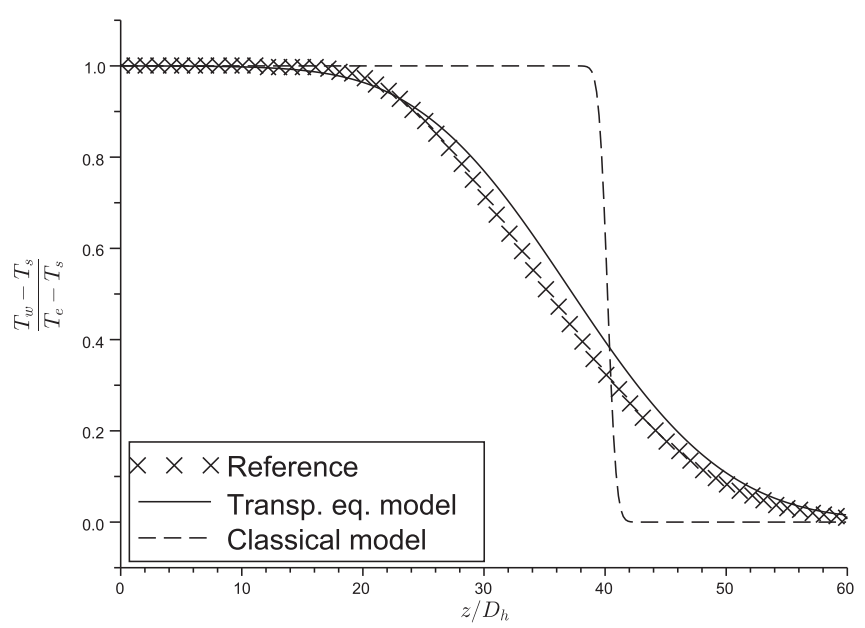

(f) Circular pipe $t_{3}=40 D_{h} /\left\langle\bar{u}_{z}\right\rangle_{f}$

Fig. 6. Wall temperature during the crossing of a temperature jump in laminar adiabatic flows in plane channels and circular pipes (see Table 3 ), at 3 different times and for $R e=175$ and $\operatorname{Pr}=1.48$. Comparison between three wall temperature profiles: classical (Eq. (86)), transport equation model (Eq. (84)) and reference results coming from spatially averaged CFD fine scale simulations are shown. Wall temperature profiles are scaled with inlet $\left(T_{e}\right)$ and outlet $\left(T_{s}\right)$ temperature values. 


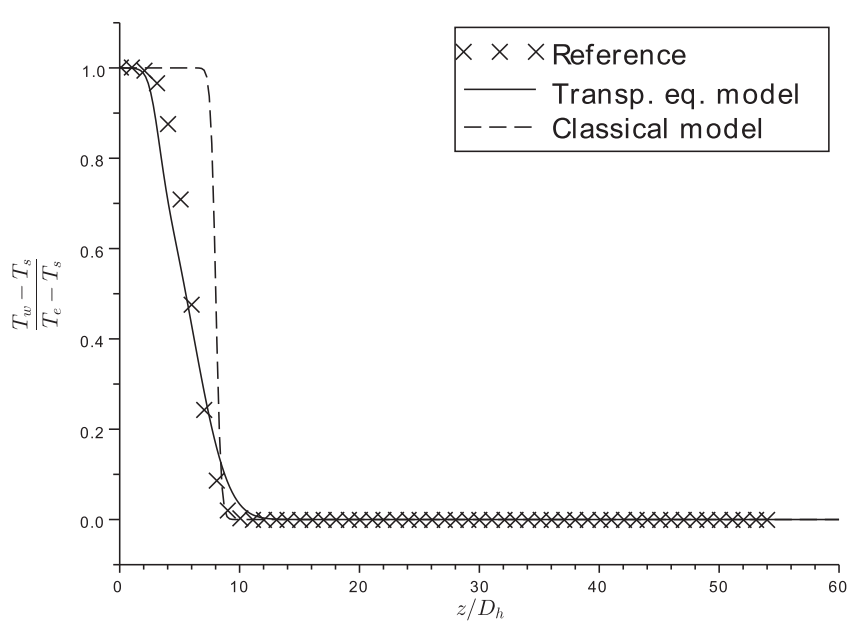

(a) Plane channel $t_{1}=8\left\langle\bar{u}_{z}\right\rangle_{f} / D_{h}$

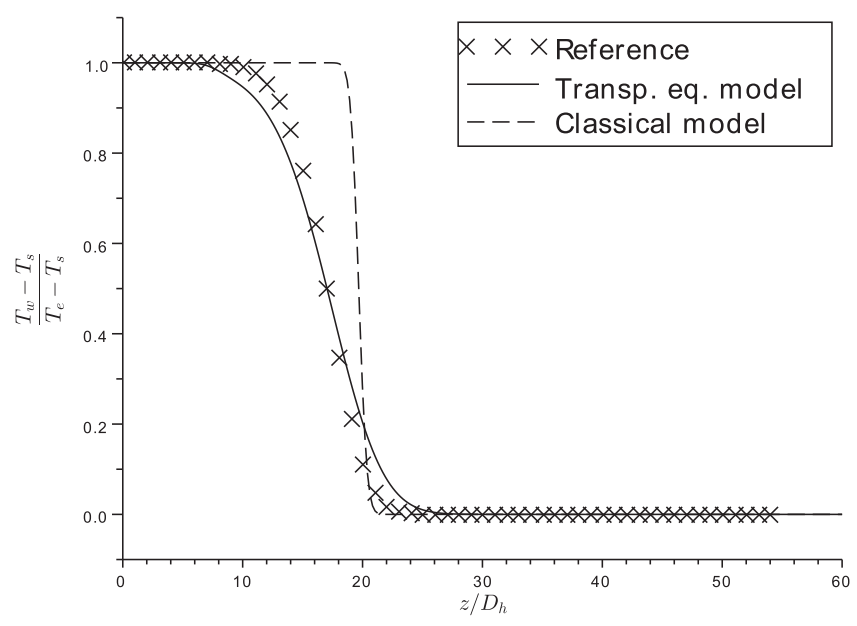

(c) Plane channel $t_{2}=20\left\langle\bar{u}_{z}\right\rangle_{f} / D_{h}$

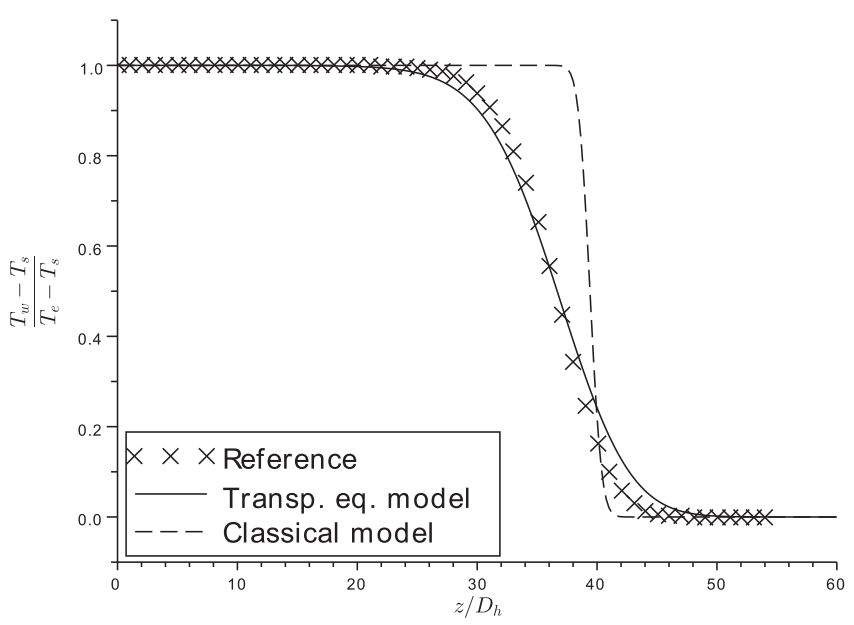

(e) Plane channel $t_{3}=40\left\langle\bar{u}_{z}\right\rangle_{f} / D_{h}$

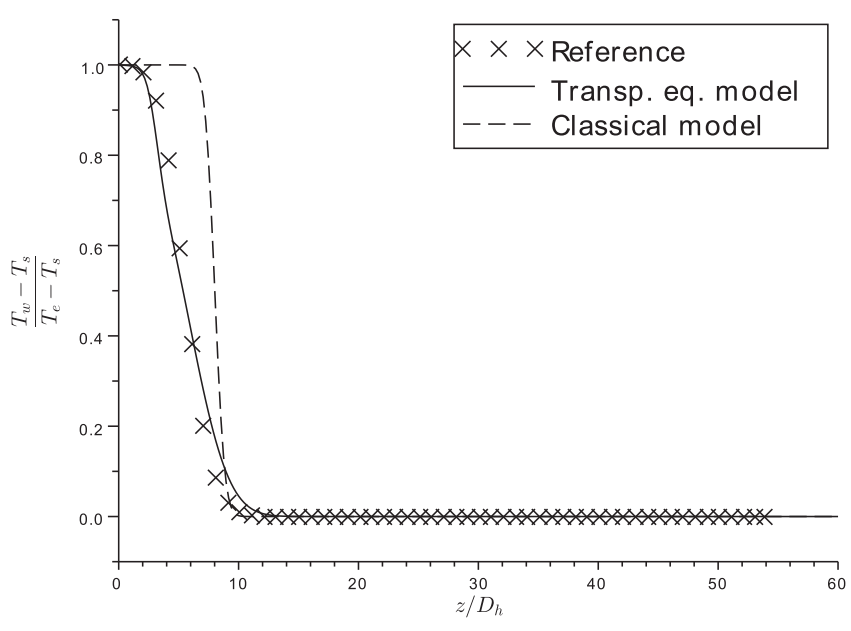

(b) Circular pipe $t_{1}=8\left\langle\bar{u}_{z}\right\rangle_{f} / D_{h}$

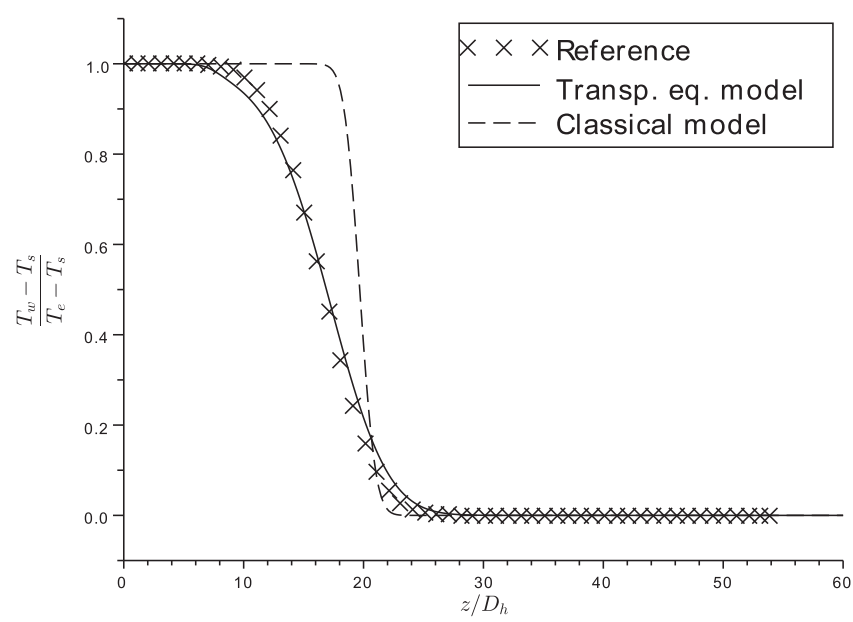

(d) Circular pipe $t_{2}=20\left\langle\bar{u}_{z}\right\rangle_{f} / D_{h}$

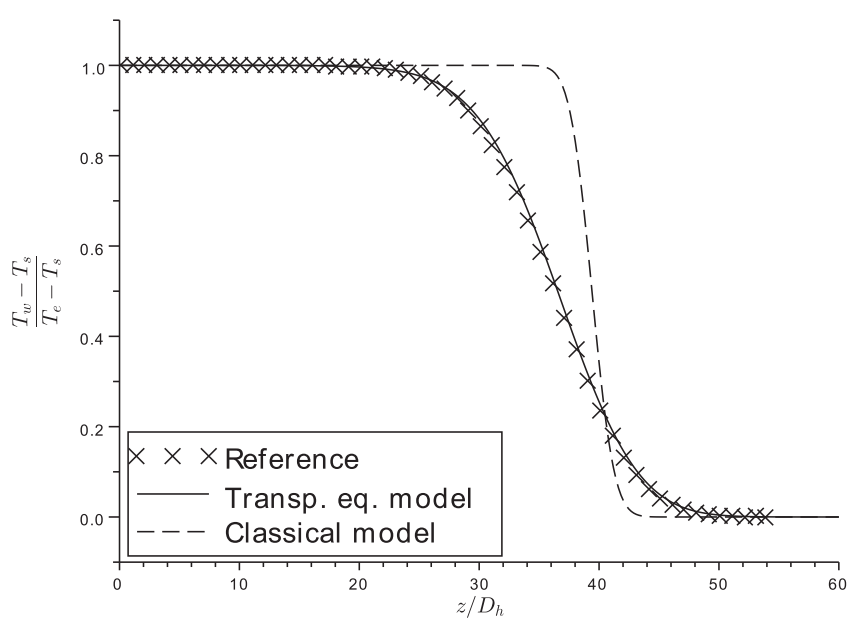

(f) Circular pipe $t_{3}=40\left\langle\bar{u}_{z}\right\rangle_{f} / D_{h}$

Fig. 7. Wall temperature during the crossing of a temperature jump in turbulent adiabatic flows in plane channels and circular pipes (see Table 3 ), at 3 different times and for $R e=15000$ and $P r=0.74$. Comparison between three wall temperature profiles: classical (Eq. (86)), transport equation model (Eq. (84)) and reference results coming from spatially averaged CFD fine scale simulations are shown. Wall temperature profiles are scaled with inlet $\left(T_{e}\right)$ and outlet $\left(T_{s}\right)$ temperature values. 


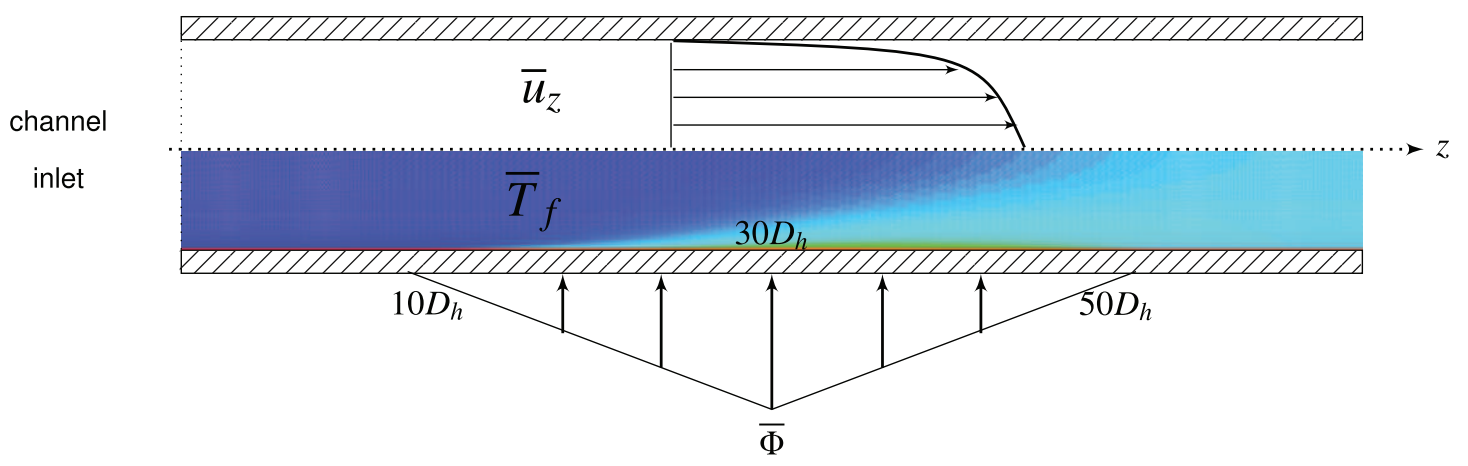

Fig. 8. Description of the wall temperature model test-case: steady flows with piecewise linear wall heat flux.

Table 4

Validation test-cases: steady flows with non-uniform wall heat flux. Acronym "CP" stands for plane channel and "Tu" for circular pipes.

\begin{tabular}{lll}
\hline & $R e$ & $\operatorname{Pr}$ \\
\hline$C P_{3}$ & 175 & 2.96 \\
$C P_{4}$ & $1.5 \times 10^{4}$ & 0.74 \\
$\mathrm{Tu}_{3}$ & 175 & 2.96 \\
$\mathrm{Tu}_{4}$ & $1.5 \times 10^{4}$ & 0.74 \\
$\mathrm{Tu}_{5}$ & 175 & 1.48 \\
$\mathrm{Tu}_{6}$ & $1.5 \times 10^{4}$ & 1.48 \\
\hline
\end{tabular}

Two types of configurations are simulated in order to highlight the model features:

1. the spreading of temperature jump by Taylor dispersion in adiabatic pipes (that kind of configurations allows to study the effects of the mean temperature gradient on wall temperature);

2. non-uniformly heated steady flows in which the effects of wall heat fluxes combined with Taylor dispersion can be observed.

\subsection{Adiabatic flows - Taylor dispersion}

We analyze the spreading of a temperature jump induced by Taylor dispersion in an adiabatic channel. This case is analogous to the case presented by Taylor $[17,18]$ except that we focus on the wall temperature. This type of flows is of great interest for industrial purposes since it is representative of the thermal shock configuration. Flows are at constant mass flow rate along the axis of the pipe, denoted $z$. The pipe is $L=60 D_{h}$ long. Initially, the fluid temperature is uniform over the pipe $T(z, t=0)=T_{s}$. The fluid temperature at the inlet, i.e. at $z=0$, is uniform across the section. At $t=t_{0}$, we increase the inlet temperature, so that $T\left(z=0, t>t_{0}\right)=T_{e}>T_{s}$. A description of the flows is shown by Fig. 3. Reynolds and Prandtl numbers hereafter considered are given in Table 3. This type of flows is representative of fast transient configurations with stiff mean temperature gradients.

For adiabatic flows, only passive dispersion contribution acts. Classical models (86), that do not account for dispersion, predict

$T_{w}=T_{B}$.

In our modeling framework, $T_{B}$ is given by (see Eq. (30))

$T_{B}=\left\langle\bar{T}_{f}\right\rangle_{f}+\frac{\left\langle\delta \bar{u} \delta \bar{T}_{f}\right\rangle_{f}}{\langle\bar{u}\rangle_{f}}$.

First of all, let us present some phenomenological results. Fig. 4 shows how dispersion acts to spread not only the averaged temperature profile, but also the bulk temperature profile. Three bulk temperature profiles are presented: the "classical" (Eq. (86)), the "dispersive" (see Eq. (9) and Drouin et al. [6]) and the "reference" bulk temperature profiles. This figure shows that the classical model only advects the stiff thermal front while the dispersive model predicts that the front is spread by dispersion. Part of the thermal jump, advected by central streamlines, progresses faster in pipes while in boundary layers, where fluid velocity is smaller, the thermal front progresses more slowly. It is clear on Fig. 4 that even bulk temperature prediction is strongly improved when accounting for dispersion flux. For the same test case, we present on Fig. 5 the bulk, averaged and wall temperatures coming from the proposed transport equation model. One can see that, as expected, wall temperature increases later than the others. Due to velocity heterogeneity across pipe section, flow is warmer in the central region than in wall region during the transient: the flow is thermally out of equilibrium. Before to be seen by the wall region, the temperature jump has to be transported from the warm central streamlines to the wall vicinity streamlines. In laminar flows, this transverse transport is only due to molecular thermal diffusion. The higher the Pr number is the longer the delay between bulk temperature increase and wall temperature increase is. In turbulent flows, turbulence amplifies the apparent diffusivity of the flow and this delay is shorter.

Figs. 6 and 7 present a comparison between classical (Eq. (86) and $T_{w}=T_{B}$ ), transport equation model (Eq. (84)) and reference results coming from the spatial average of fine scale CFD simulations. Since classical model does not account for dispersion, it only advects a stiff thermal front. On the contrary, the transport equation model, coupled with macroscopic temperature model, exhibits widely spread wall temperature profiles over tens of hydraulic diameters. With respect to classical model, temperature increases earlier (due to dispersion modeling), but much more slowly. For instance, in circular pipe at $t=40 D_{h} /\langle\bar{u}\rangle_{f}$ (Fig. 6 (f)), wall temperature at $60 D_{h}$ starts to increase and the asymptotic wall temperature is achieved at $16 D_{h}$. In other words, the thermal front spreads over $44 D_{h}$ while, calculated according to the classical model, the thermal front should be concentrated on $4 D_{h}$. Improvement of wall temperature profiles is striking in both laminar and turbulent regimes.

\subsection{Non-uniform wall heat flux}

In this section, we study the case of a steady flow with very heterogeneous wall heating. This configuration activates both passive and active dispersion contributions. The fluid flows along the axis of the pipe, denoted $z$. The pipe is $L=60 D_{h}$ long. The fluid temperature at $z=0$ is uniform over the section $T(z=0, t)=T_{0}$. For simplicity convenience, we impose triangular profiles for the wall heat flux (see Fig. 8):

$$
\Phi= \begin{cases}0 & \text { si } z<10 D_{h} \text { ou } z>50 D_{h}, \\ \Phi_{\max } \frac{z-10 D_{h}}{20 D_{h}} & \text { si } 10 D_{h}<z<30 D_{h}, \\ \Phi_{\max } \frac{50 D_{h}-z}{20 D_{h}} & \text { si } 30 D_{h}<z<50 D_{h} .\end{cases}
$$




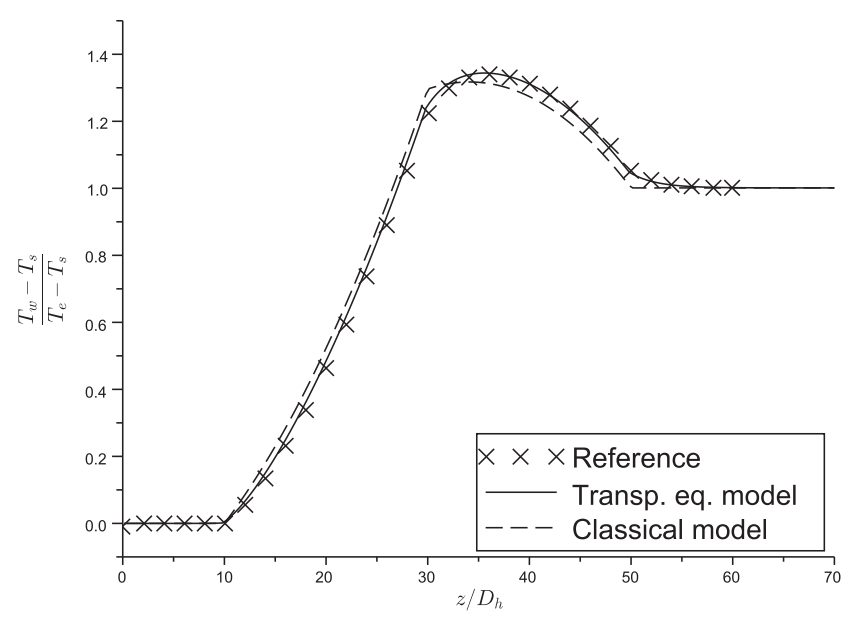

(a) $C P_{3}$ : plane channel, $R e=175, \operatorname{Pr}=2.96$.

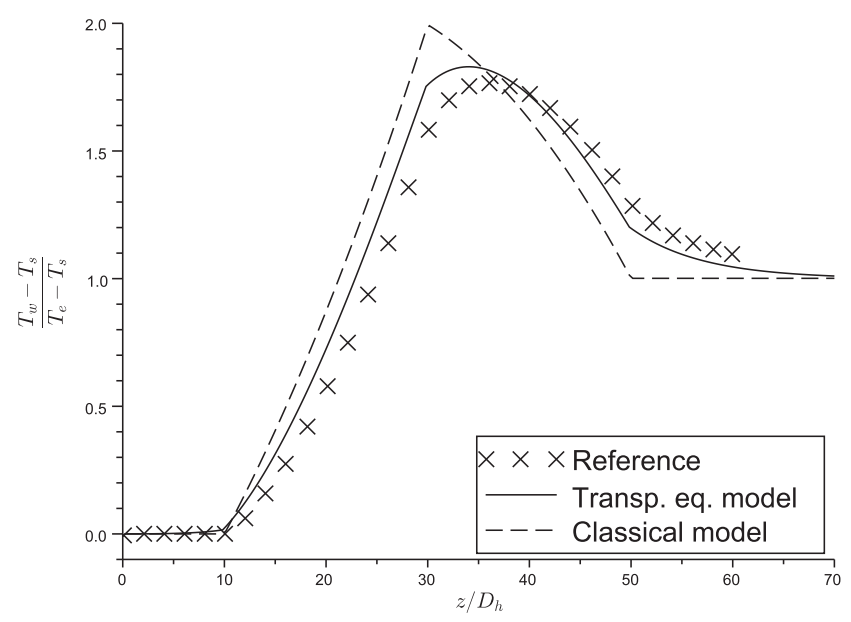

(c) $T u_{3}$ : circular pipe, $\operatorname{Re}=175, \operatorname{Pr}=2.96$.

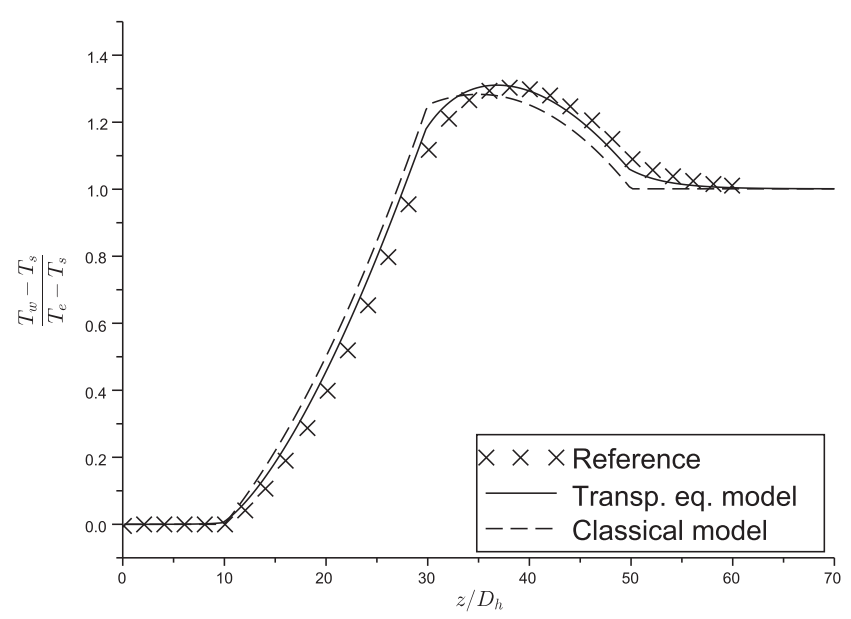

(e) $T u_{5}$ : circular pipe, $\operatorname{Re}=175, \operatorname{Pr}=1.48$.

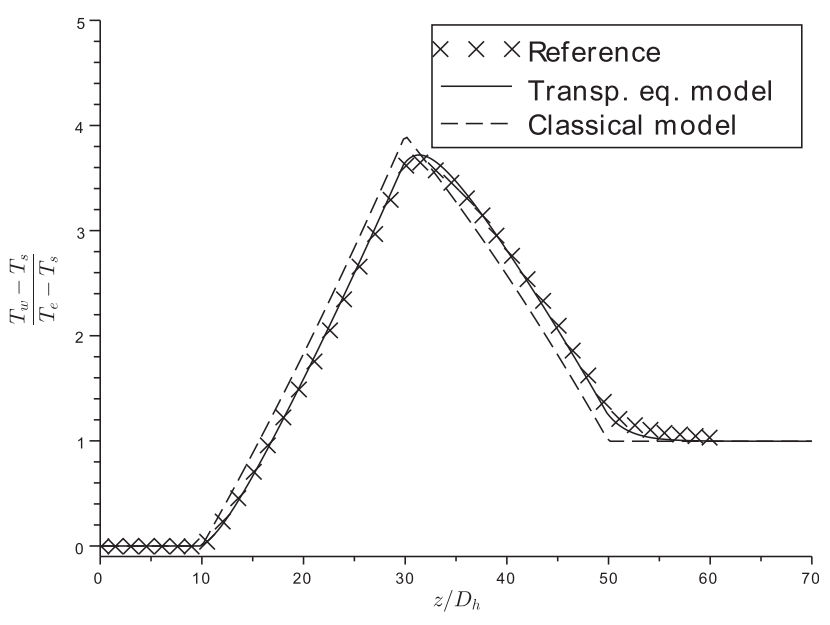

(b) $C P_{4}$ : plane channel, $R e=15000, \operatorname{Pr}=0.74$.

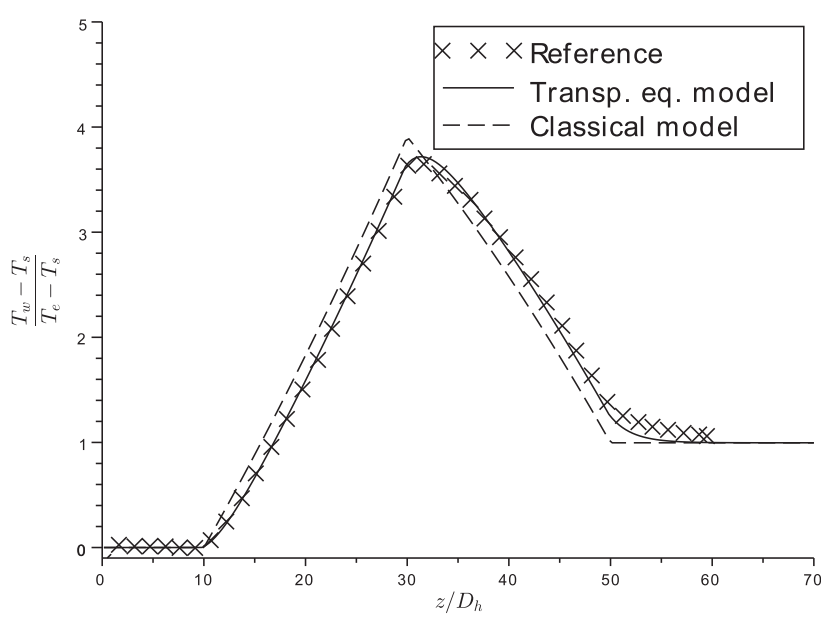

(d) $T u_{4}$ : circular pipe, $\operatorname{Re}=15000, \operatorname{Pr}=0.74$.

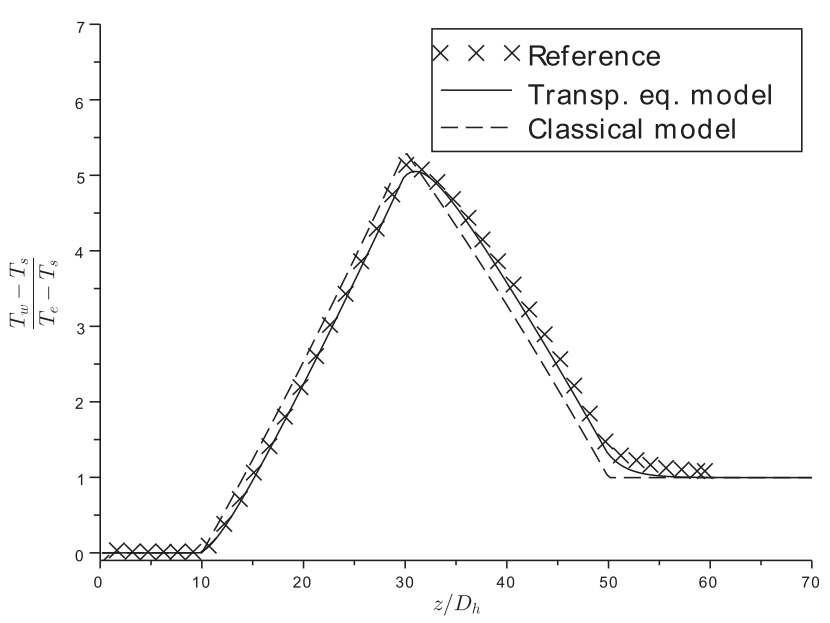

(f) $T u_{6}$ : circular pipe, $R e=15000, \operatorname{Pr}=1.48$.

Fig. 9. Non-uniformly heated flows in pipes: Non-dimensioned wall temperature profiles. Comparison between three wall temperature profiles: classical (Eq. (86)), transport equation model (Eq. (84)) and reference results coming from spatially averaged CFD fine scale simulations are shown. Wall temperature profiles are scaled with inlet $\left(T_{e}\right)$ and outlet $\left(T_{s}\right)$ temperature values. 
Thermal flux in various test cases presented here is each time tuned in order to induce the same temperature increase whatever the mass flux. The cases studied below are summarized in Table 4 .

A comparison between results obtained with classical heat exchange model, transport equation model and reference results is shown by Fig. 9 for all flow conditions of Table 4. On the left side of profiles, i.e. in the region where heat flux linearly increases, all calculations predict an increase of wall temperature. The classical wall temperature model predicts wall temperature profiles perfectly aligned with wall heat flux. Nevertheless, wall temperatures calculated thanks to fine scale simulations all exhibit a slight shift downstream with respect to the heat flux profile. The higher the Pr number is, the larger the shift is. This shift comes from the thermal unbalance between outer and inner parts of the boundary layer: the outer region of the boundary layer is colder than if it was at thermal equilibrium and thus cools the wall region. This complex phenomenology is well predicted by the transport equation model for both laminar and turbulent regimes. One has to notice that, with the proposed model, the maximum of wall temperature profiles is located downstream the location of the maximum of the heat flux. This result is also consistent with fine scale simulation results.

On the right hand side, i.e. in the region where heat flux linearly decreases, the classical model predicts a decrease of wall temperature until heat flux vanishes. Then, it predicts $T_{w}=T_{B}$. On the contrary, the transport equation model predicts that wall temperature remains higher than bulk temperature even downstream the point where wall heat flux vanishes. This prediction is consistent with fine scale simulation results. This effect was expected. Indeed, as boundary layer at the end of the heating region is still warmer than bulk flow, it continues to exchange some heat with wall after the heating region. Once again, it is an effect of the thermal unbalance between various regions of the flow. Amplitude of this effect appears to be still underestimated for turbulent regime. Nevertheless, amplitude of this phenomenon as well as $\operatorname{Pr}$ number sensitivity are well predicted for laminar regime. Within the turbulent regime, no sensitivity to $\operatorname{Pr}$ number is observed (see Fig. 9(d) and (f)). This result was expected since transverse diffusion is due to turbulence and not to molecular diffusion for this flow regime.

The capability to predict the spatial shift between bulk and wall temperature variations for heterogeneous heat fluxes in an important feature of the proposed model. If one considers a very heterogeneous heating, say a ponctual heating device, our model shall be able to predict not only the average temperature, but also wall temperature downstream. Hence, it could be used, for instance, to define the proper thermocouple locations for experimental devices design without performing expensive fine scale simulations.

\section{Conclusion}

In this paper, we have attempted to connect dispersion modeling and heat transfer prediction for both laminar and turbulent regimes in stratified porous media. To this aim, we have extended, as a first step, the closure problem exposed in Drouin et al. [6] to wall temperature prediction. We use temperature deviation modeling in order to connect wall to mean temperatures for well established pipe flows. For flows with large thermal unbalance, i.e. for fast transients and for huge heat flux gradients, wall temperature encounters stiff temperature gradients (especially within turbulent regime) and is thus mainly controlled by boundary conditions. Consequently, the scale separation assumption poorly applies on wall temperature and the algebraic model corresponding to the asymptotic limit for regular flows does not apply.

In order to increase the application domain of such an approach, we avoid this limitation by deriving a transport equation for wall temperature. We introduce a two layer approximation that allows us to distinguish bulk and boundary layer length and time scales. This transport equation is easily closed from the algebraic model written for the limit case of smooth flows (slow transients and regular gradients flow conditions).

Implemented in a hydro code, this transport equation model has been successfully compared with fine scale CFD simulations. Improvement in wall temperature prediction is striking. The delay between wall and bulk flow conditions is well recovered, especially for the thermal shock configuration. Furthermore, in the adiabatic region downstream a very stiff heat flux gradient, we show that it is possible to predict the heating of walls by hot boundary layers. Model derived in this paper might be used for design purposes of heat exchangers with accuracy similar to CFD calculations and for a much lower computational cost.

\section{References}

[1] D. Bestion, The physical closure laws in the CATHARE code, Nucl. Eng. Des. 1249 (3) (1990) 229-245.

[2] R.G. Carbonell, S. Whitaker, Dispersion in pulsed systems - II: Theoretical developments for passive dispersion in porous media, Chem. Eng. Sci. 38 (11) (1983) 1795-1802.

[3] K.Y. Chien, Prediction of channel and boundary layer flows with a lowReynolds number turbulence model, AIAA J. 20 (1) (1982) 33-38.

[4] A.P. Colburn, A method of correlating forced convection heat transfer data and a comparison with fluid friction, Trans. AIChE 19 (1933) 174-210.

[5] F.W. Dittus, L.M.K. Boelter, Heat transfer in automobile radiators of the tabular type, Univ. Calif. Publ. Eng. 2 (1930) 443-461.

[6] M. Drouin, O. Grégoire, O. Simonin, A. Chanoine, Macroscopic modeling of thermal dispersion for turbulent flows in channels, Int. J. Heat Mass Transfer 53 (2010) 2206-2217.

[7] M. Drouin, Modlisation des coulements turbulents anisothermes en milieux macroporeux par une approche de double filtrage, $\mathrm{PhD}$ thesis, Université de Toulouse, France, 2010.

[8] O. Grégoire, Remarques sur la turbulence permettant de retrouver quantitativement ou qualitativement un certain nombre de résultats expérimentaux: le point de vue en 2003, Technical Report, SFME/LETR/RT/ 03-013/A, CEA, 2003.

[9] O. Grégoire, Établissement formel d'un modèle diphasique macroscopique à 6 équations, lien avec le modèle macroscopique à 4 équations', Technical Report, CEA-R-6185, CEA, 2008.

[10] S. Kakac, R.K. Shah, W. Aung, Handbook of Single-Phase Convective Heat Transfer, John Wiley and Sons, 1987.

[11] A. Nakayama, F. Kuwahara, A macroscale turbulence model for flows in a porous medium, J. Fluids Eng. 121 (1999) 427-433.

[12] M.H.J. Pedras, M.J.S. De Lemos, Macroscopic turbulence modeling for incompressible flow through undeformable porous media, Int. J. Heat Mass Transfer 44 (2001) 1081-1093.

[13] F. Pinson, O. Grégoire, M. Quintard, M. Prat, O. Simonin, Modeling of turbulent heat transfer and thermal dispersion for flows in flat plate heat exchangers, Int. J. Heat Mass Transfer 50 (2007) 1500-1515.

[14] M. Quintard, S. Whitaker, Transport in ordered and disordered porous media I: The cellular average and the use of weighting functions, Transport Porous Med. 14 (1994) 163-177.

[15] M. Quintard, S. Whitaker, Transport in ordered and disordered porous media II: Generalized volume averaging, Transport Porous Med. 14 (1994) 179-206.

[16] H. Reichardt, ZAMM - Journal of Applied Mathematics and Mechanics/ Zeitschrift fr Angewandte Mathematik und Mechanik (1951) 208-219.

[17] G. Taylor, Dispersion of solute matter in solvent flowing slowly through a tube, Proc. Roy. Soc. Lond. A 219 (1953) 183-203.

[18] G. Taylor, The dispersion of matter in turbulent flow through a pipe, Proc. Roy. Soc. Lond. A 223 (1954) 446-468.

[19] I. Toumi, A. Bergeron, D. Gallo, E. Royer, D. Caruge, FLICA-4: a threedimensional two-phase flow computer code with advanced numerical methods for nuclear applications, Nucl. Eng. Des. 200 (1-2) (2000) 139-155.

[20] S. Whitaker, Diffusion and dispersion in porous media, AIChE 13 (3) (1967) 420-427.

[21] S. Whitaker, Theory and Applications of Transport in Porous Media: The Method of Volume Averaging, Kluwer Academic Publishers, 1999. 